\title{
Active Galactic Nuclei: Basic Physics and Main Components
}

\author{
H. Netzer
}

School of Physics and Astronomy, Tel Aviv University, Tel Aviv 69978, Israel

netzer@wise.tau.ac.il

\begin{abstract}
This review gives a brief introduction to the physics and the structure of active galactic nuclei (AGN). The main topics that are being discussed are general accretion, the structure and the spectrum of various types of accretion disks, photoionization and recombination, energy balance and gas temperature, the spectrum of ionized gas including the effect of dust, and the dynamics of ionized gas in a strong radiation field. These principles are then applied to various AGN components including the broad and the narrow line regions, the ionized X-ray gas, the torus and the central disk.
\end{abstract}

\section{Terminology, Classification and Abbreviations}

The study of active galactic nuclei (AGN) has produced many new terms and abbreviations. Only the most common ones will be used in this review, e.g., the narrow line region (NLR), the broad line region (BLR), the highly ionized gas (HIG) and the black hole (BH). I shall avoid, as much as possible, using names such as Seyfert 1s, Seyfert 2s and LINERs. Instead I shall refer to type-I AGN (those objects showing broad, strong optical-UV emission lines in their spectrum), and type-II AGN (those showing prominent narrow emission lines, very faint, if any, broad lines, and a large X-ray obscuring column).

\section{Black Holes}

Black holes (BHs) of all sizes are very common in the universe. This has been known for many years. However, the understanding that most galaxies, especially early type galaxies, contain massive BHs is more recent. New studies compare BH mass and evolution in high redshift quasars to the evolution of early galaxies. It seems that massive BHs, as large as $10^{9} M_{\odot}$, have already been present at $z \simeq 6$ [1]. Some of these sources seem to accrete matter at a very high rate. This raises important questions about the growth rate and the formation mechanism of those BHs. Any BH and accretion disk model, like the one presented below, must be compatible with such observation.

The basic properties of BHs can be expressed using their gravitational $\left(r_{g}\right)$ and Schwarzschild $\left(r_{s}\right)$ radii,

H. Netzer: Active Galactic Nuclei: Basic Physics and Main Components, Lect. Notes Phys. 693, 1-38 (2006) 


$$
r_{g}=\frac{G M}{c^{2}}, r_{s}=2 r_{g} .
$$

We can also define the $\mathrm{BH}$ angular momentum $s$

$$
s \sim I \Omega \simeq M r_{g}^{2} \frac{v}{r} \simeq M r_{g} c,
$$

where $\Omega$ is the angular velocity, and the $\mathrm{BH}$ specific angular momentum (angular momentum per unit mass),

$$
s / M \equiv a c \sim r_{g} c \sim 5 \times 10^{23} M_{8} \mathrm{~cm}^{2} \mathrm{~s}^{-1},
$$

where $M_{8}$ is the mass in units of $10^{8} M_{\odot}$. Considering massive BHs in galactic nuclei, we note that the typical specific angular momentum on galactic scales (e.g. $r=1 \mathrm{kpc}, v=300 \mathrm{~km} \mathrm{~s}^{-1}$ ) is many orders of magnitude larger than $a c$ in (3). Thus, for BHs to grow substantially over time scales typical of galactic evolution, there must be an efficient mechanism to get rid of the excess angular momentum of the accreted gas (in order to bring it to the center). On large scales, BH growth depends on mechanisms such as galaxy collision and merging and bar instability that are capable of bringing far away gas into the vicinity of the $\mathrm{BH}$.

Gas particles very close to the center, at $r_{g} \sim 1000$, are subjected to different types of forces and are not necessarily accreted onto the $\mathrm{BH}$. The understanding of their motion, temperature and emission requires a discussion of those mechanisms including the balance between gravity and radiation pressure force, the general process of spherical accretion and the properties of thin and thick accretion disks.

\subsection{The Eddington Luminosity}

Assume a central source of mass M emitting monochromatic luminosity $L_{\nu}$. The radiation pressure force acting on gas particles at a distance $r$ is given by

$$
f_{\text {rad }}=\frac{N_{e} \sigma_{T}}{4 \pi r^{2} c} \int_{0}^{\infty} L_{\nu} d \nu=\frac{N_{e} L \sigma_{T}}{4 \pi r^{2} c} L,
$$

where $L$ is the total luminosity, $N_{e}$ is the electron density and $\sigma_{T}$ the Thomson cross section. The gravitational force is

$$
f_{g}=\frac{G M \mu m_{p} N_{e}}{r^{2}}
$$

where $\mu$ is the mean molecular weight (mean number of protons and neutrons per electron; about 1.17 for a fully ionized solar composition gas). Spherical accretion of fully ionized gas can proceed as long as $f_{g}>f_{\text {rad }}$. The requirement $f_{\text {rad }}=f_{g}$ leads to the so-called "Eddington luminosity",

$$
L_{E d d}=\frac{4 \pi c G M \mu m_{p}}{\sigma_{T}} \simeq 1.5 \times 10^{38}\left(M / M_{\odot}\right) \operatorname{erg~s}^{-1},
$$


which is the maximum luminosity allowed for objects that are powered by steady state accretion. This definition of $L_{E d d}$ takes into account only one source of opacity, Compton scattering. This is the case for fully ionized plasma. More realistic situations may involve partly neutral gas and hence much larger opacity. Thus, the effective $L_{E d d}$ can be significantly smaller than the value defined in (6).

Given the definition of $L_{E d d}$, and the accretion rate $\dot{M}=L / \eta c^{2}$, where $\eta$ is the efficiency of converting gravitational potential energy to electromagnetic radiation, we can define several other useful quantities. Thus, the "Eddington accretion rate", $\dot{M}_{E d d}$, is the accretion rate required to produce $L_{E d d}$,

$$
\dot{M}_{E d d}=\frac{L_{E d d}}{\eta c^{2}} \simeq 3 M_{8}\left[\frac{\eta}{0.1}\right]^{-1} M_{\odot} y^{-1}
$$

The "Eddington time", $t_{E d d}$, is the typical time associated with this accretion rate and is given by

$$
t_{E d d}=\frac{M}{\dot{M}_{E d d}} \simeq 4 \times 10^{8} \eta \mathrm{yr} .
$$

This terminology allows us to express the relative accretion rate (accretion rate per unit mass of the $\mathrm{BH}$ ) in the following way:

$$
\frac{L}{L_{E d d}} \propto \frac{\dot{M}}{\dot{M}_{E d d}} \propto \frac{\dot{M}}{M} .
$$

The normalized Eddington accretion rate, $\dot{m}=\dot{M} / \dot{M}_{E d d}$, is used in various articles. Unfortunately, this is done in an inconsistent way since in some articles it includes the efficiency factor $\eta$ (like in here) while in others $\eta$ is not included (i.e. $\dot{m}=c^{2} \dot{M} / L_{E d d}$ ).

\subsection{Spherical Accretion}

The simplest possible case involves spherical accretion (also known as "Bondi accretion"). In this case we assume that accretion starts from a large radius where the gas is at rest. We follow the gas radial motion under the combined force of gravity and radiation pressure. Detailed calculations [2], show that the optical depth of such gas is very large. For example, if the gas is completely ionized, its electron scattering optical depth is

$$
\tau_{e} \simeq \frac{\dot{M} / \dot{M}_{E d d}}{\left[r / r_{g}\right]^{1 / 2}},
$$

which, for a critical accretion rate $\left(\dot{M}=\dot{M}_{E d d}\right)$ with inflow at the free fall velocity, gives a Compton depth of order unity. The radiation conversion efficiency $(\eta)$ in spherical accretion is very small. The reason is that, in such 
accretion, most of the radiation is emitted by two body collisional processes in the ionized plasma. Such processes are relatively inefficient compared with the release of gravitational energy. The result is that most of the gravitational energy is advected into the central object and the radiated luminosity is only a small fraction of the released gravitational energy. A good estimate is

$$
\frac{L}{L_{E d d}} \simeq 10^{-4}\left[\frac{\dot{M}}{\dot{M}_{E d d}}\right]^{2} .
$$

\section{Accretion Disks}

The most efficient accretion processes are associated with accretion disks. Such systems are naturally formed by infalling gas that sinks into the central plane while retaining most of its angular momentum. Such disks, if dense and thick enough, can provide the necessary mechanism to transfer angular momentum out and to allow gas infall into the vicinity of the BH [3]. There are several types of accretion disks with different shapes and properties. The most important ones are discussed here in general terms.

\subsection{Basic Disk Parameters}

First we consider optically thick geometrically thin accretion disks. Such systems are formed and maintained for a large range of accretion rates, $10^{-4} \lesssim L / L_{E d d} \lesssim 0.3$. The important physical parameters of such systems are the angular momentum of the material in the disk at radius $r, s(r)$, the net torque associated with this angular momentum, $N(r)$, and the radial drift velocity of the gas, $v_{r}[4,5]$. In general all these quantities can change as a function of time. Here we limit the discussion to time independent, stationary disks.

Consider first the angular momentum of a ring of radius $r$ with a total (time independent) mass $m$,

$$
s=m v_{\phi} r,
$$

where $v_{\phi}$ is the azimuthal velocity. For most cases of interest $v_{r} \ll v_{\phi}$ and $v_{\phi} \simeq v_{K}$, where $v_{K}$ is the Keplerian velocity. The associated Keplerian angular velocity is

$$
\Omega_{K}=\frac{v_{K}}{r}=\left[\frac{G M}{r^{3}}\right]^{1 / 2} .
$$

The radial mass flow rate of a stationary disk, $\dot{M}$, is constant and is given by

$$
\dot{M}=-2 \pi r \Sigma v_{r}
$$


Here $\Sigma$ is the surface mass density of the disk $\left(\mathrm{cm}^{2}\right)$, which is also time independent and is given by

$$
\Sigma=2 \rho H(r),
$$

where $\rho$ is the mean density and $H(r)$ the scale height (see below) at a radius $r$.

Given these assumptions we can find the net torque (i.e. the torque between two adjacent rings) by considering conservation of angular momentum. This means that the angular momentum carried inward across radius $r$ minus the angular momentum transported outward by the torque exerted on the disk outside of that radius, is constant. This requires that

$$
N(r)=\dot{M}[G M r]^{1 / 2}+\text { const. }
$$

Thin accretion disks are defined by those properties as well as their inner $\left(r_{i n}\right)$ and outer $\left(r_{\text {out }}\right)$ radii. The innermost radius is where the assumption of bound Keplerian motion breaks down. This is normally referred to as the "last stable orbit" and is a function of the angular momentum of the BH. For non-rotating BHs $r_{i n}=6 r_{g}$ while for a maximally rotating Kerr BH $r_{i n}=1-1.2 r_{g}$. Regarding the outer radius, $r_{\text {out }}$, this is not well defined and depends on the pressure and the gravity at large distances (see Subsect. 3.5). The various disk parameters are shown in Fig. 1.

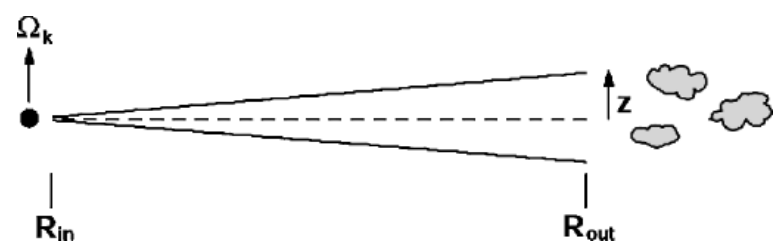

Fig. 1. Definition of the basic disk parameters (see text). The disk breaks into self gravitating blobs beyond the self-gravity radius, $r_{\text {out }}$ (courtesy of K. Sharon)

\subsection{Energy and Luminosity}

The energy release in thin accretion disks is determined by the loss of gravitational energy of the inward going material and the work done by the torque that results in outward loss of angular momentum. The first of these can be written as

$$
d L_{g}=-d\left[\dot{M} \frac{G M}{2 r}\right]
$$

and the second as 


$$
d L_{N}=-d\left(N \Omega_{K}\right)=-d\left[\dot{M} \frac{G M}{r}\right],
$$

where $L_{g}$ and $L_{N}$ are the luminosities associated with those terms. Combining the two and differentiating with respect to $r$ we obtain an expression for the energy release at various radii,

$$
\frac{d L}{d r}=\frac{3 G M \dot{M}}{2 r^{2}}\left[1-\left(\frac{r_{i n}}{r}\right)^{1 / 2}\right],
$$

where the second term on the right takes into account the innermost radius of the disk, $r_{i n}$. Simple integration over parts of the disk far from its center shows that $L_{r} \propto G M \dot{M} / r$. The total radiated power, obtained by integrating over the entire disk, in the non-relativistic limit, is

$$
L=\frac{1}{2} \frac{G M \dot{M}}{r_{i n}} .
$$

This is half the total available power meaning that, neglecting relativistic corrections, the matter at $r_{i n}$ still retains a kinetic energy which is half the potential energy it has lost.

Relativistic corrections are important for thin accretion disks since much of the radiation is released close to $r_{i n}$. They change the geometric correction factor, $1-\left(\frac{r_{i n}}{r}\right)^{1 / 2}$, in a significant way, especially for rapidly rotating BHs. Including those terms one finds that for a Schwarzschild $\mathrm{BH}, 50 \%$ of the energy is emitted between 6 and about $30 r_{g}$ while for extreme Kerr BHs, the limits are even closer. It is thought that in thin AGN accretion disks, much of the ionizing radiation is released at radii of order $\sim 10 r_{g}[6]$.

\subsection{Emissivity and Temperature}

The expression derived above for $d L / d r$ enables us to obtain the emissivity per unit area of the disk,

$$
D(r)=\frac{3 G M \dot{M}}{8 \pi r^{3}}\left[1-\left(\frac{r_{i n}}{r}\right)^{1 / 2}\right] .
$$

Assuming local emission as a perfect blackbody with a temperature $\mathrm{T}(D=$ $\sigma T^{4}$ ), we get an expression for the disk temperature at all radii,

$$
T(r)=\left(\frac{3 G M \dot{M}}{8 \sigma \pi r^{3}}\left[1-\left(\frac{r_{i n}}{r}\right)^{1 / 2}\right]\right)^{1 / 4}
$$

Expressing the radius in units of $r_{g}$ and the accretion rate in normalized units of $\dot{M} / \dot{M}_{E d d}$ (i.e. for a given $L / L_{E d d}$ ) enable us to compare accretion disks around various mass BHs. This gives 


$$
T(r) \propto M_{8}^{-1 / 4}\left[\frac{\dot{M}}{\dot{M}_{E d d}}\right]^{1 / 4}\left[\frac{r}{r_{g}}\right]^{-3 / 4} .
$$

For scaling purposes we note that in a thin accretion disk around a $\mathrm{BH}$ with $M_{8}=1$, the temperature is $T\left(6 r_{g}\right) \sim 10^{5} \mathrm{~K}$. Such disks emit most of their energy in the UV part of the spectrum. In a similar way, the maximum blackbody temperature associated with thin accretion disks around stellarsize BHs is about $10^{7} \mathrm{~K}$, with most of the energy emitted in the X-ray part of the spectrum. Thus, accretion disks around massive BHs are much cooler than stellar size accretion disks. Also, for standard geometrically thin optically thick disks, $T(r) \propto r^{-3 / 4}$.

\subsection{Viscosity}

The only variable which is not yet fully defined is the radial velocity $v_{r}$. This is determined by the viscosity of the disk which in turn depends on the microphysics (atomic or molecular processes) of the disk material, the turbulence in the disk, the magnetic fields strength and structure, etc $[4,5,7,8,9]$. It is customary to define the viscosity stress, $\nu$, such that

$$
\nu=\alpha v_{s} H
$$

where $H$ is the scale height of the disk (to be defined later) and the parameter $\alpha$ includes all unknowns regarding the disk microphysics. This results in a family of disk models referred to as " $\alpha$ disk models". The simplest form assumes the same $\alpha$ for all parts of the disk. The radial velocity in $\alpha$ disks is found by combining the accretion rate (14) and the torque (16) equations with the definition of $\nu$. This gives

$$
v_{r}=\frac{3 \nu}{2 r} \ll v_{s} .
$$

Thus, the radial inflow is subsonic.

We can combine the various parameters in a different way to obtain

$$
\nu \rho \Omega_{K}=\alpha P,
$$

where $P$ is the total pressure. In this prescription the viscosity depends on the pressure. There are various variants of this prescription where $\alpha$ is proportional to the total pressure, the gas pressure, or some combination of the two.

The above definitions allow us to write an expression for the important "viscous time scale", $t_{v i s}$, which is the radial drift time from radius $r$ to $r_{i n}$,

$$
t_{v i s} \simeq \frac{r}{v_{r}} \simeq \frac{2 r^{2}}{3 \nu} .
$$

For steady state AGN disks this time is very long (many years). 


\subsection{Disk Geometry}

Accretion disks can be thin, slim or thick, depending on the mass accretion rate. This can be seen by considering the vertical component of the $\mathrm{BH}$ gravity, $g_{z}$, at some distance $r$,

$$
g_{z}=\frac{G M}{r^{2}} \sin \theta \simeq \frac{G M}{r^{3}} z=\Omega_{K}^{2} z,
$$

and the hydrostatic equation

$$
\frac{d P}{d z}=-\rho g_{z}=-\rho \Omega_{K}^{2} z
$$

where

$$
P=P_{g}+P_{\text {rad }}=\frac{\rho k T}{\mu m_{p}}+\frac{1}{3} a T^{4} .
$$

Assume first that the total pressure is determined by the gas pressure, $P=P_{g}$. In this case, $P=\rho v_{s}^{2}$, where $v_{s}$ is the sound speed. The hydrostatic equation is

$$
\frac{d P}{d z}=v_{s}^{2} \frac{d \rho}{d z}=-\rho \Omega_{K}^{2} z
$$

with the simple solution

$$
\rho=\rho_{0} \exp \left[-\frac{z^{2}}{H^{2}}\right] ; H \simeq \frac{v_{s}}{\Omega_{K}},
$$

where $\rho_{0}$ is the mid-plane density of the disk. Thus

$$
\frac{H}{r} \simeq \frac{v_{s}}{v_{K}}
$$

and the disk is geometrically thin since $v_{s} \ll v_{K}$.

Next, consider the self gravity of the disk in the $z$ direction,

$$
g_{z}^{\text {self }}=2 \pi G \Sigma=2 \pi G(2 \rho z),
$$

and compare it with the vertical component of the central gravitational field, $g_{z}$. We expect the disk to break into small fragments at those radii where $g_{z}^{\text {self }}>g_{z}$, which gives a natural outer boundary for the disk, $r_{\text {out }}$. This boundary, together with the inner boundary $r_{i n}$, and the disk scale height, $H$, specify the thin disk geometry (see Fig. 1).

\subsection{Slim and Thick Accretion Disks}

The situation is very different when the accretion rate, that determines the disk temperature and hence $P_{\text {rad }}$, exceeds a critical value where $P_{\text {rad }}>P_{g}$. The simple hydrostatic solution of (32) is no longer valid and a more accurate 
treatment gives much larger $H / r$. Such systems are sometimes called "slim" or "thick" accretion disks, depending on their exact geometry. Calculations show that AGN disks are dominated by radiation pressure [6]. However, for $L / L_{E d d} \lesssim 0.3$ the hydrostatic solution gives $H / r$ which is small enough for the disk to be considered "thin". This is no longer the case for larger accretion rate where more accurate solutions must be considered.

Large accretion rate disks can be inefficient emitters since the accretion (inflow) time scale can be shorter than the time it takes for radiation to diffuse to the surface of the disk. The photons in such disks can become trapped in the accretion flow leading to low radiation efficiency [10].

It is speculated that some AGN (e.g. narrow line Seyfert 1 galaxies, NLS1s, and very high luminosity quasars) are accreting at rates that are close or even exceed the Eddington accretion rate. These are the most likely candidates to host slim or thick accretion disks. A derivation of the mass of such systems based on the emitted radiation can lead to erroneous results, due to the processes discussed here.

\subsection{The Spectrum of Accretion Disks}

The expressions obtained above for the emissivity and local temperature of thin accretion disks can be combined to derive the local emitted monochromatic luminosity,

$$
d L_{\nu}=2 \pi r\left(\pi B_{\nu}\right) d r
$$

and the total emitted monochromatic luminosity,

$$
L_{\nu}=\int_{r_{\text {in }}}^{r_{\text {out }}} d L_{\nu}=\frac{4 \pi^{2} h \nu^{3}}{c^{2}} \int_{r_{\text {in }}}^{r_{\text {out }}} \frac{r d r}{\exp (h \nu / k T)-1} \propto \nu^{1 / 3},
$$

where the last step involves the substitutions $x=h \nu / k T, T \propto r^{-3 / 4}$ and the assumption that $x_{\text {out }} \gg x_{\text {in }}$. This simple $\nu^{1 / 3}$ dependence holds over a limited energy band because of the physical limits of the disk. The outer disk boundary imposes a minimal disk temperature. Thus, at low frequencies the spectrum resembles a blackbody with $T=T\left(r_{\text {out }}\right)$. This gives, at low frequencies, $L_{\nu} \propto \nu^{2}$. At the high frequency end, $L_{\nu}$ drops exponentially with a functional dependence corresponding to the maximum disk temperature (emitted at a radius slightly larger than $r_{i n}$ ). A schematic representation of the spectrum of such ideal disks is shown in Fig. 2.

\subsection{Complex Disks and ADAFs}

The application of the thin-disk theory to AGN is problematic for several reasons. Most important, such disks cannot explain the entire emitted spectral energy distribution (SED) since much of AGN energy is emitted in the $\mathrm{X}$-ray band yet the maximum disk temperature cannot exceed few $\times 10^{5} \mathrm{~K}$. 


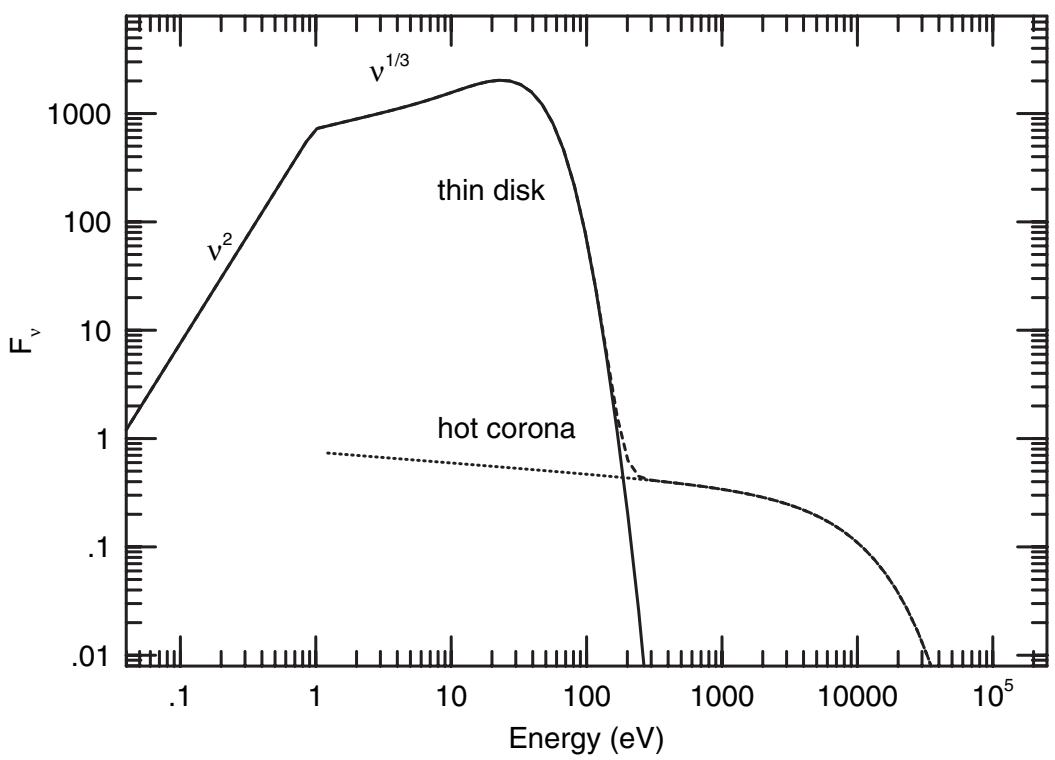

Fig. 2. Schematic representation of a disk-corona spectrum assuming a geometrically thin optically thick accretion disk with $T_{\max }=10^{5} \mathrm{~K}$ and an optically thin corona with $T_{\text {cor }}=10^{8} \mathrm{~K}$

It is therefore plausible to assume an additional hot medium, in the vicinity of the disk, where more energetic photons are emitted. An appealing idea is that of a hot dilute gas where the soft disk-emitted photons are upscattered, via Compton scattering, to their observed X-ray energy. This process (Comptonization) can also explain the observed shape of the X-ray continuum which resembles a simple power-law over a large range of frequencies. The condition for the hot medium is to have a non-negligible Compton depth.

Accretion through the outer layers of the disk can result in a large energy dissipation and a sharp temperature rise. The outer skin expands and the disk develops a hot corona [11]. Such a structure can considerably modify the emitted spectrum. In particular, hard photons that are created (or scattered) in the corona can either escape the disk-corona structure or else be absorbed by the cool disk and hence change its energy balance. Likewise, soft disk photons can be absorbed by the corona in some parts or freely escape in other parts, depending on the geometry. All these effects are difficult to calculate.

A combined solution of the hydrostatic and radiative transfer equations for the disk-corona configuration is complicated and involves some ad-hoc assumptions [10]. A simplified treatment could include the assumption of an optically thin corona with a small scale height (i.e. geometrically thin disk-corona system). The corona temperature in this case is determined by the fraction of the total accretion power released in the corona. Even a very small fraction, such as $1 \%$ of the total accretion rate, can result in very high 


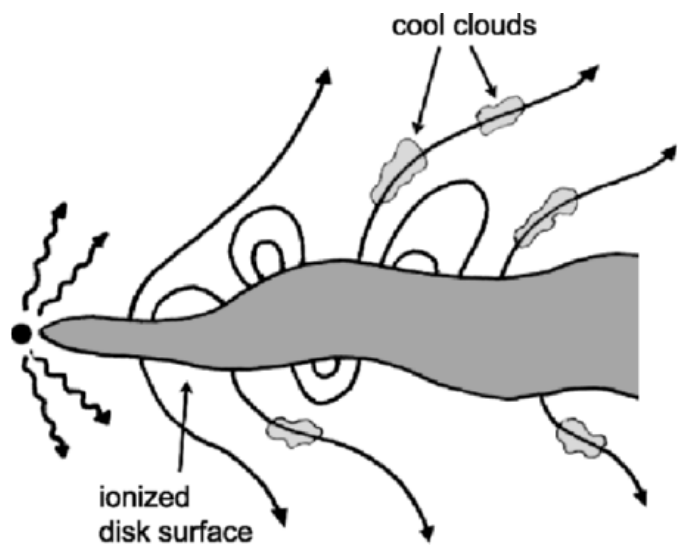

Fig. 3. Complex magnetic disks can include a non-uniform geometry like the one shown, schematically, in this diagram. Cool clouds can be flown from the surface of the disk along the magnetic field lines. Such gas is exposed to the central radiation field at some height above the disk surface (courtesy of K. Sharon)

temperatures, $\sim 10^{8} \mathrm{~K}$. Such coronae radiate through free-free processes and their emission can dominate the spectrum at X-ray energies. As explained, depending on their Compton depth, they can also up-scatter the soft Xray photons which results in additional X-ray flux. A schematic spectrum a corona dominated by free-free X-ray emission is shown in Fig. 2.

Real disks are likely to be very complex. In particular, strong magnetic fields can strongly influence the disk viscosity and may drive massive winds from the surface. A possible (schematic and highly simplified) configuration is shown in Fig. 3. Numerical calculations are needed to solve for the structure and the spectrum of such systems.

Very low accretion rate disks are interesting and important for other reasons, not necessarily related to AGN. Low accretion rate results in weak coupling between the ions (mostly protons) and the electrons which will lead to two different temperatures for the two types of particles. While protons carry most of the gravitational energy, cooling, and hence electromagnetic energy release is mostly due to electrons. In such systems, the heavier protons are advected into the $\mathrm{BH}$ taking with them much of the gravitational energy. Such advection dominated accretion flows (ADAFs) are very hot yet very inefficient emitters. It is speculated that some AGN disks can fluctuate between the states of high accretion rate and ADAF, depending on the fuel supply to the center.

\subsection{Spectral Energy Distribution}

The spectral energy distribution (SED) of AGN is complex, with significant variations between different energy bands and different objects. Figure 4 


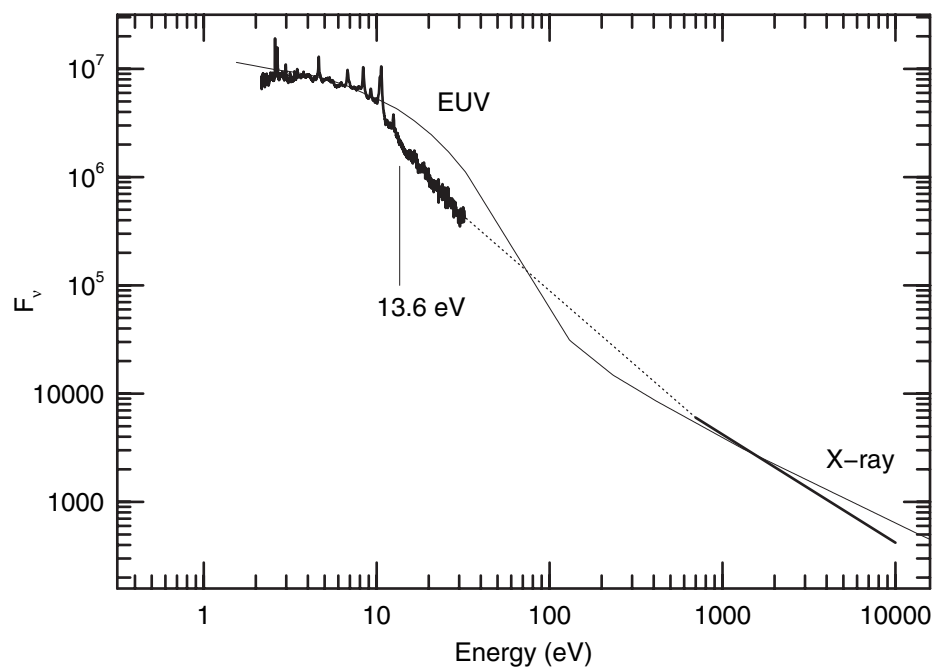

Fig. 4. Observed SED (thick solid lines connected with a thin dotted line) compared with a theoretical SED composed of a spectrum of a thin accretion disk around a $3 \times 10^{9} M_{\odot} \mathrm{BH}$ with $L / L_{E d d}=0.1$. The theoretical spectrum has an additional component of an X-ray power-law. Note the strong "blue bump" (energy excess close to and beyond the Lyman limit) predicted by this model

shows a composite SED made of the combined spectrum of several hundred AGN in the 1-20 eV range and a "typical" X-ray powerlaw continuum.

The observed SED in Fig. 4 looks different than the schematic disk spectrum in Fig. 2. However, there are good reasons to believe that the optical-UV continuum of AGN is dominated by disk emission. First, real accretion disks do not emit as simple blackbodies. The run of density and temperature with distance from the mid-plane of real disks result in a thin atmosphere where conditions are quite different from thermal equilibrium. Integrating over the various parts of such disks results in a spectrum which is considerably different from the schematic $\nu^{1 / 3}$ dependence and is closer in shape to $L_{\nu} \propto \nu^{-s}$, with $s \sim 0.5$ in the optical-UV band. Second, the infrared part of the SED $(\lambda \geq 1 \mu \mathrm{m})$ is dominated by non-disk processes (thermal emission by dust and nonthermal synchrotron emission). This obscures the part of the spectrum where pure disks are predicted to show $L_{\nu} \propto \nu^{2}$. A theoretical spectrum that includes several of those effects is shown in Fig. 4 where it is compared with the composite observed spectrum.

\section{Physical Processes in AGN Gas}

Dilute ionized gas in AGN is found from very close to the central BH, at $r \simeq$ $1000 r_{g}$, to distances of several $\mathrm{kpc}[12,13]$. This gas is exposed to the strong 
central radiation field and its properties are well described by considering photoionization and recombination and neglecting most other ionization and excitation processes. Notable exceptions are regions of violent star formation where mechanical heating and ionization, due to super-novae explosions and fast stellar winds, are major sources of energy input to the gas. The spectrum of such regions is typical of thermal plasma although photoionization can be important too.

The following is a brief account of the principles and the more important processes necessary to analyze and model the spectrum of active galaxies. The main assumption is that the gas density is low, $N_{H}<10^{12} \mathrm{~cm}^{-3}$, such that collisional transitions among excited states are of secondary importance for most ions (but not for hydrogen and helium) [12].

\subsection{Photoionization and Radiative Recombination}

Consider gas at a distance $r$ from a point source of total luminosity $L$ and monochromatic luminosity $L_{\nu}$. The fractional ionization of ion $X$ is determined by the ionization rate per particle due to flux reaching the location of the ion, $I_{X}$, and by the recombination rate per particle, $R_{X}$. These rates are given by

$$
I_{X}=\int_{\nu_{X}}^{\infty} \frac{\left(L_{\nu} / h \nu\right) \sigma_{\nu} e^{-\tau_{\nu}}}{4 \pi r^{2}} d \nu,
$$

and

$$
R_{X}=\alpha_{X}(T) N_{e}
$$

where $\nu_{X}$ is the (ground state) threshold ionization frequency for ion $X$, $\alpha_{X}(T)$ is the summed recombination coefficient for the ion, and $\tau_{\nu}$ the optical depth. The time dependent fractional ionization is obtained from the solution of

$$
\frac{d N_{X}}{d t}=-N_{X}\left[I_{X}+R_{X-1}\right]+N_{X-1} I_{X-1}+N_{X+1} R_{X},
$$

where $N_{X}$ is the number density of ion $X$. All other ionization and recombination processes have been neglected at this stage. The steady state solution is

$$
\frac{N_{X+1}}{N_{X}}=\frac{I_{X}}{R_{X}}
$$

which represents $n$ equations for $n+1$ ions. The additional equation is

$$
\Sigma N_{X}=N_{\text {element }}
$$

where $N_{\text {element }}$ is the total abundance of the element in question.

The above set of equations leads to the definition of two important time scales: the recombination time, $1 / R_{X}$, and the ionization time, $1 / I_{X}$. The first gives the typical time required for ion $X+1$ to recombine to ion $X$, when the radiation source is turned off. The second time scale is the time required for 
$X$ to get ionized to $X+1$ after a large increase in the ionizing flux. We note that the recombination time is density dependent but the ionization time is not. Thus, following a time dependent recombination provides a way to probe the local conditions while following a time-dependent ionization enables to set a limit on the distance $r$.

The recombination and ionization time scales can be quite different, depending on the radiation field and the gas density. Moreover, the coupling between the various stages of ionization suggests that, under various conditions, the fractional abundance of ion $X$ may change very slightly, or remains almost constant, despite large changes in the radiation field. This leads to somewhat different definitions of the recombination time involving positive contributions (recombination of $X^{+1}$ ) as well as negative contributions (recombination to $X^{-1}$ ). Such expressions can be found in various other reviews [14]. Perhaps more important are the global recombination and ionization time scales which are the times required for the entire atmosphere to undergo a significant change in the level of ionization. Both are given roughly by $1 / R_{X}$ where $X$ is the most abundant ion of the element in question.

\subsection{Additional Atomic Processes}

While photoionization from the ground level and radiative recombination following cascade are the dominant processes in AGN ionized gas, other processes must be considered too. The most important ones are:

Dielectronic recombination: These are normally divided into "low temperature dielectronic recombination", due to $\Delta n=0$ transitions, where $n$ is the energy level under question, and "high temperature dielectronic recombination", $\Delta n=1$ transitions. The first are important for many elements at $T_{e}=1-3 \times 10^{4} \mathrm{~K}$ and the second for hot $\left(T_{e} \sim 10^{6-7} \mathrm{~K}\right)$ plasma.

Auger ionization: Ionization from inner shells due to absorption of high energy X-ray photons is important for ions with three or more electrons. The process couples non-adjacent stages of ionization and results in X-ray fluorescence lines that are commonly observed in the spectrum of AGN.

Heating and ionization by secondary electrons: This process is important for gas with a very low level of ionization which is exposed to a large flux of high energy photons. The energetic electrons ejected due to the absorption of such photons can collide with neutral atoms (mostly hydrogen) and cause additional ionization before they are thermalized. They can also collisionally excite some lines, such as Ly $\alpha$.

Charge exchange: Charge exchange collisions between various ions can be very fast, leading to significant changes in the level of ionization under some favorable conditions.

Collisional ionization and three body recombination: These processes are negligible for most ions for temperatures below few $\times 10^{5} \mathrm{~K}$. Collisional 
ionization dominates the ionization of the gas in starburst regions. Both processes are important in high density large optical depth clouds where they can dominate the population of the high-n levels of hydrogen and helium.

\subsection{Thermal Balance}

The thermal structure of the gas is governed by a set of equations that involves energy terms rather than ionization and recombination terms. These will be noted by $H$ for heating and $C$ for cooling. In AGN gas, the most important heating is photoionization (bound-free) heating which, for ion $X$, is given by

$$
H_{b f, X}=N_{X} \int_{\nu_{X}}^{\infty} \frac{L_{\nu} \sigma_{\nu} e^{-\tau_{\nu}}}{4 \pi r^{2}} d \nu .
$$

In this equation we sum over the absorbed energy while in the photoionization equation (37) we summed over the number of ionizing photons. Similarly, an important cooling term is "recombination cooling" given by

$$
C_{b f, X}=N_{X+1} N_{e} \alpha_{X}(T)\left(h \nu_{1, \infty}+\left\langle h \nu_{f r e e}\right\rangle\right)
$$

where $h \nu_{1, \infty}$ is the ionization energy from the ground level of ion $X$ and $\left\langle h \nu_{\text {free }}\right\rangle$ is the mean energy of the free electron (of order $k T_{e}$ ).

The above heating and cooling terms are different from those used in most text books where only the energy of the free electrons is considered [15]. In that case the term $L_{\nu}$ in the heating integral should be replaced by $\left(L_{\nu} / h \nu\right)\left(h \nu-h \nu_{X}\right)$ and the recombination equation does not include the $h \nu_{1, \infty}$ term. All other heating and cooling terms can be computed with either of the two equivalent treatments provided they are used consistently when calculating the heating-cooling balance.

In many cases, the most important cooling term is due to line cooling. This involves solving for the time dependent statistical equilibrium equations which result in the time dependent level population and the net cooling (net line emission). This is illustrated here for a two-level system and only three processes: recombination with a rate $\alpha_{\text {eff }}$, collisional excitation and deexcitation with rates $q_{12}$ and $q_{21}$ respectively, and line (but no continuum) photon emission and absorption. The time dependent equations in this case are

$$
\frac{d n_{2}}{d t}=n_{1} q_{12}-n_{2}\left(A_{21} \beta_{21}+q_{21}\right)+N_{X+1} N_{e} \alpha_{\text {eff }}
$$

and

$$
n_{1}+n_{2}=N_{X},
$$

where the abundance of ion $X, N_{X}$, is also time-dependent (see (39)). Potentially important processes that are neglected in this simplified treatment are dielectronic recombination, collisional ionization, three body recombination, photoionization from both levels and continuum pumping (see below). 
The term $\beta_{21}$ in (44) is the "local escape probability", i.e. the probability of line photons to leave the point where they are created without being absorbed by 1-2 transitions in a different location. This simplified escape probability treatment replaces the more rigorous, full radiative transfer solution. It enables a full local solution but neglects radiative coupling of different locations in the gas. Detailed radiative calculations show that a reasonable approximation for $\beta$ is $\beta=1 /\left(1+b \tau_{12}\right)$, where $\tau_{12}$ is the optical depth between the two levels and $b$ is a constant of order unity [12].

Having solved for $n_{1}$ and $n_{2}$, we can now express the line emissivity, $\epsilon_{21}$, which is also the bound-bound (bb) cooling term, $C_{b b}$, as follows:

$$
C_{b b}=\epsilon_{21}=n_{2} \beta_{21} A_{21} h \nu_{21} .
$$

The general energy equation is $H=C$, where $H=\Sigma_{i} H_{i}$ and $C=\Sigma_{i} C_{i}$ are sums over all heating and cooling terms. The solution of this equation is used to obtain the kinetic temperature of the gas. This is relatively simple in steady state, where the level population and ionization fractions are not time dependent. The main complications are due to radiative transfer effects that couple level populations to the gas temperature through collisional excitation and de-excitation processes.

\section{Mechanical Heating of AGN Gas}

The general energetics and the physical conditions in AGN gas are controlled primarily by the central radiation field. This is in contrast to different environments, such as star-forming regions, where mechanical heating is the dominant heating source. There have been several suggestions that parts of the gas in the nucleus is affected by collisional processes, e.g. small-scale jets can transfer a large amount of mechanical energy into a very small region, producing strong shocks and bright emission knots typical of collisionally excited plasma. However, general considerations suggest that such processes cannot be globally important in the type of environment considered here.

The mechanical energy produced by a shock of velocity $v_{\text {shock }}$ is roughly $m_{\text {shock }} v_{\text {shock }}^{2}$ where $m_{\text {shock }}$ is the mass of the flowing material. Equivalently, if $m_{a c c}$ is the mass of the gas accreted by the BH then the energy associated with this process is about $\eta m_{a c c} c^{2}$, where $\eta$ is the accretion efficiency factor. The observed velocity of much of the AGN line emitting gas is of order $500 \mathrm{~km} \mathrm{~s}^{-1}$ or larger and, for accretion disks around massive BHs, $\eta \sim 0.1$. Thus the ratio of the two energy producing processes is approximately $10^{5}\left(m_{a c c} / m_{\text {shock }}\right)$. For shock excitation to be energetically significant, the amount of flowing shocking gas must exceed, by many orders of magnitude, the amount of accreted material. This seems to be in contradiction with known properties of AGN. 


\subsection{The Spectrum of Ionized AGN Gas}

\section{Ionization Parameters}

Given photoionization-recombination equilibrium, one can define an "ionization parameter" which is proportional to the level of ionization of the gas. One possible definition is

$$
U=\int_{E_{1}}^{E_{2}} \frac{\left(L_{E} / E\right) d E}{4 \pi r^{2} c N_{\mathrm{H}}},
$$

where $c$ is the speed of light (introduced to make $U$ dimensionless) and $E_{1}$ and $E_{2}$ are energy boundaries to define the photon flux. $U$ defined in this way gives a good indication for the level of ionization provided $E_{1}$ is close to the minimum energy required to ionize the most "important" (coolingwise) ions and elements. For gas that is ionized by soft-UV radiation, $E_{1}$ should be chosen such that it is related to the UV ionizing field, e.g. the energy of the hydrogen Lyman limit. An appropriate name in this case is $U$ (hydrogen). Similarly, a suitable choice for gas whose level of ionization is dominated by a strong soft X-ray source is $E_{1}$ inside that energy range. Such gas can be transparent to UV photons and $U$ (hydrogen) is not a good indicator of its level of ionization. A suitable choice in this case is $E_{1}=0.54$ $\mathrm{keV}$, corresponding to the K-shell threshold ionization of oxygen, the most important emitting and absorbing element under such conditions. A suitable name is $U$ (oxygen).

There are other, somewhat different definitions of the ionization parameter that are used in various publications. A common one, $\xi$, is defined by the energy flux rather than the photon flux,

$$
\xi=\int_{13.6 \mathrm{eV}}^{13.6 \mathrm{keV}} \frac{L_{E}}{r^{2} N_{H}} d E .
$$

This ionization parameter has been used in many X-ray related papers.

Using the "right" photon flux ionization parameter, we normally find that $U=10^{-1}-10^{-2}$ corresponds to gas that produces strong emission lines in the energy range under question. For example, $U$ (oxygen) $=0.1$ results in strong $0.5-3 \mathrm{keV}$ emission lines. A much smaller $U$ results in more neutral gas, with very little $\mathrm{X}$-ray emission, and a much larger $U$ in gas that is so highly ionized that line emission is insignificant.

Table 1 gives a list of several ionization parameters that are useful in various situations and are commonly used in AGN research. The right column of the table allows a simple relative scaling of those ionization parameters for the case of $L_{E} \propto E^{-1.3}$.

\section{Line and Continuum Emitting Processes}

The most important continuum emitting processes are due to bound-free transitions. The signature of such processes in the optical-UV band are the 
Table 1. Various ionization parameters used in the literature

\begin{tabular}{llll}
\hline Ionization Parameter & $E_{1}$ & $E_{2}$ & $\begin{array}{l}\text { Conversion Factors, } \\
L_{E} \propto E^{-1.3}\end{array}$ \\
\hline$U$ (hydrogen) & $13.6 \mathrm{eV}$ & $\infty$ & 1000 \\
$U$ (helium) & $54.4 \mathrm{eV}$ & $\infty$ & 165 \\
$U$ (X-ray) & $0.1 \mathrm{keV}$ & $10 \mathrm{keV}$ & 73.3 \\
$U$ (oxygen) & $0.54 \mathrm{keV}$ & $10 \mathrm{keV}$ & 8.2 \\
$\xi$ & $13.6 \mathrm{eV}$ & $13.6 \mathrm{keV}$ & 31.1 \\
\hline
\end{tabular}

bound-free transitions to levels $n=1$ and $n=2$ of hydrogen and helium. In the X-ray band the observationally important processes are bound-free transitions to the ground levels of H-like and He-like carbon, nitrogen, oxygen, neon, magnesium and silicon. Free-free emission is important at radio frequencies, in a low temperature gas, and at hard X-ray energies, in a high temperature plasma.

Line emission (bound-bound) transitions dominate the heating-cooling balance in low ionization $(U$ (hydrogen $\sim 0.01$ ) photoionized AGN gas. The most intense transitions are due to forbidden lines, in low density gas, and due to recombination and collisionally ionized permitted lines, in high density gas. For highly ionized X-ray gas $(U$ (oxygen) $\sim 0.01)$, most cooling is via boundfree transitions because the lowest accessible levels are at energies that are much larger than $k T$. This gas is dominated by permitted, intercombination and forbidden recombination lines. Starburst X-ray gas, which is sometimes found in AGN, is characterized by higher temperatures and lower densities. The most intense lines are produced via collisional excitation of $n=2$ and $n=3$ levels.

Several additional processes that were not discussed so far result in line emission and absorption. Here we comment on two of those.

Continuum fluorescence: This process, sometimes referred to as "photoexcitation" or "continuum pumping", is the result of populating low lying levels by absorbing the incident continuum in various resonance transitions. This increases the level population and the line emission but the effect on the global energy balance is negligible, especially in low density environment, since heating and cooling almost exactly balance.

The increase or decrease in the observed line intensity, due to this process, is geometry dependent. For example, in a static spherical atmosphere, or in a static spherical thin shell, where the point continuum source is in the center, the process can be considered as pure scattering and emission is completely canceled out by absorption. In a moving spherical atmosphere, the emitted photons are Doppler shifted relative to the gas rest frame, and absorption and emission appear at different energies. This results in both emission and absorption with the same equivalent widths (EWs). In cases 
of incomplete covering, absorption is stronger than emission (in terms of the measured EW). Finally, in optically thick lines, the absorption and emission optical depths are not necessarily the same which can affect the emitted line intensity.

Continuum fluorescence in the X-ray band can also excite inner shell transitions. In principle, this is similar to absorption by resonance lines except that some of these transitions can leave the ion in an autoionized stage which can affect the ionization balance.

Line fluorescence: Photoionization of K-shell electrons can lead to line fluorescence, following the ejection of an Auger electron. The process is important at X-ray energies where lines of this type (most notably iron $\mathrm{K} \alpha$ lines in the $6.4-7.0 \mathrm{keV}$ energy range) are occasionally seen. The fluorescence efficiency ("fluorescence yield") depends on $Z^{3}$ and is roughly 0.3 for iron. Thus, the most abundant elements, like oxygen, do not produce strong fluorescence lines, despite the order of magnitude difference in abundance between iron and oxygen. This difference can partly be compensated by the larger incident flux around $0.54 \mathrm{keV}$ (the K-shell threshold for neutral oxygen), compared with the flux near $7.1 \mathrm{keV}$ (the K-shell threshold of neutral iron).

\subsection{Clouds, Condensations, Confinement and Winds}

The basic line emitting entity is normally referred to as a cloud. The assumption of clouds, or more generally condensations, is reasonable given the observations of galactic HII regions, and the interstellar medium, where condensations are indeed observed. It is also required by line intensity considerations, mainly the observation that the typical observed line widths, e.g. in the BLR, are similar for low and high ionization lines. The assumption of clouds is somewhat problematic since such entities must be either gravitationally bound or else confined by external pressure.

Likely candidates for self gravitating clouds are stars in the central cluster. In particular winds produced by "bloated stars" have been considered as the origin of material in the broad and the narrow line regions. It is interesting to note that "typical" NLR clouds (that are not just theoretical entities since direct observations of nearby AGN show clear condensations in the NLR) contain masses of about one $M_{\odot}$. Much lower mass clouds, containing as little as $10^{-8} M_{\odot}$, have been considered in various BLR models. Such clouds must be confined or else created and destroyed on a very short (the sound speed crossing time) time scale. For the BLR clouds, this time is of order one year.

The most likely confinement mechanisms for clouds that are not selfgravitating are either thermal confinement, by a low density hot inter-cloud medium (HIM), or confinement by magnetic fields. Confinement requires pressure equilibrium and the exact physical conditions are obtained from 
general considerations of a two-phase medium, similarly to what is done in the ISM $[16,17]$.

Consider a medium where the fractional ionization of the gas and its kinetic temperature are determined solely by the central radiation field. Suppose there are two components at the same location with very different temperatures that are noted here by "cold" and "hot". In this case,

$$
U_{\text {cold }} \propto \frac{L / c}{r^{2} N_{H}(\text { cold })} ; U_{h o t} \propto \frac{L / c}{r^{2} N_{H}(h o t)} .
$$

Dividing by the relevant temperature $\left(T_{\text {cold }}\right.$ or $\left.T_{\text {hot }}\right)$ and noting that all quantities are calculated at the same location we get

$$
\frac{U_{\text {cold }}}{T_{\text {cold }}}=\frac{U_{\text {hot }}}{T_{\text {hot }}} \propto \frac{P_{\text {rad }}}{P_{g}} .
$$

At a given distance $r, P_{\text {rad }} \propto L / r^{2}$ and is independent of the gas density. Thus, for all components at that location $\frac{U}{T} \propto P_{g}^{-1} \propto\left(N_{H} T\right)^{-1}$. This allows several different temperatures and densities for the same value of $U / T$. For the case considered here there are two possible values of $T$ for the same $U / T$. For other cases there may well be even more such solutions, all with the same $P_{g}$. Such two (or more) components can survive, side by side, in pressure equilibrium, provided they are stable against thermal perturbations. The situation is illustrated in Fig. 5 where we show the famous "S-curve", which is the photoionization equilibrium curve (sometimes referred to as the "stability curve") for such gas.

The upper branches of stability curves like the ones shown here are stable, i.e. a small deviation from the curves results in return to an adjacent stable region. Their temperature is the Compton temperature of the fully ionized gas which is determined solely by Compton heating and cooling and is given by

$$
T_{C}=\frac{h \bar{\nu}}{4 k},
$$

where $h \bar{\nu}$ is the mean photon energy weighted by the cross section. $T_{C}$ depends only on the SED and, for AGN, is $\sim 10^{7} \mathrm{~K}$. The lower branch with the positive slope is also stable and is typical of AGN photoionized gas with a characteristic temperature of $\sim 10^{4} \mathrm{~K}$. The intermediate part contains one or more regions with negative slopes. These are unstable regions where small perturbations to the right (the region where $H>C$ ) or to the left $(H<C)$ result in further removal from the stability line. Thus a two, three and even four-component medium can be formed in gas exposed to a typical AGN radiation field. Such localized components, with a well defined temperature, could be described as "clouds" or condensations.

An alternative and perhaps a more likely solution is confinement by magnetic pressure. In this case, $B^{2} / 8 \pi \geq N_{H} k T$ and the required magnetic field is of the order of one gauss for the BLR and much smaller for the NLR. 


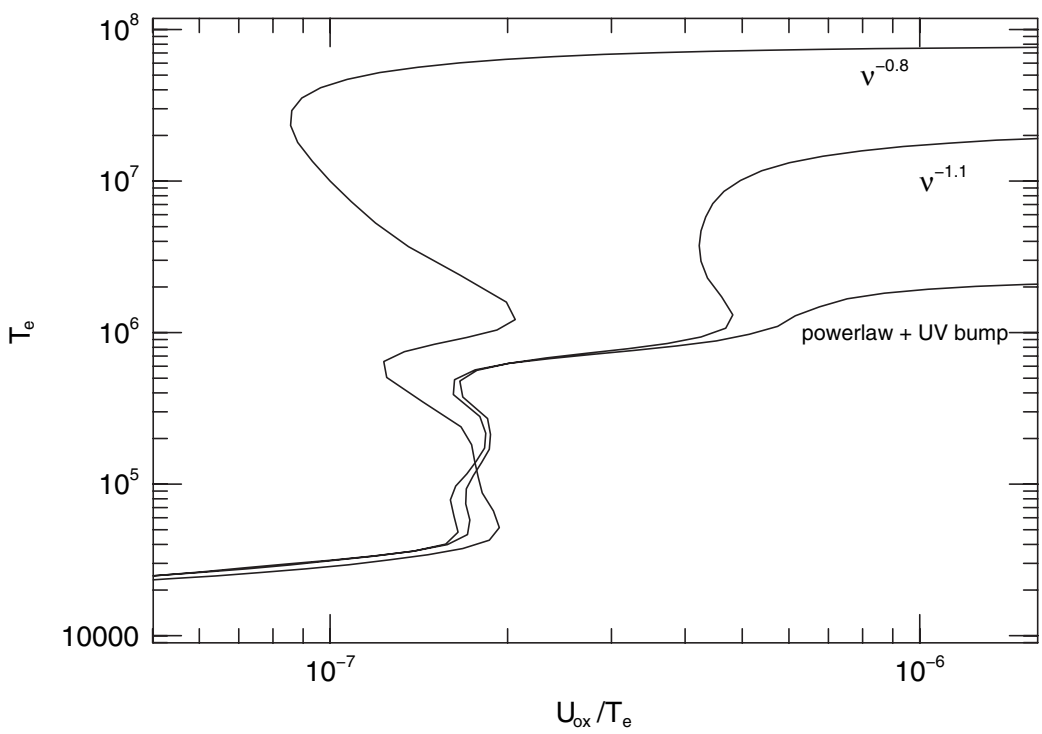

Fig. 5. Stability curves for several assumed SEDs, calculated by the author: 1 . $L_{E} \propto E^{-0.8}$. 2. $L_{E} \propto E^{-1.1}$. 3. "Typical" AGN SED with a strong blue-bump and an X-ray power-law. Note the several unstable regions (negative slope parts) that are less noticeable in the SED containing a UV bump. Such SED results in lower $T_{C}$ due to the efficient Compton cooling by the blue-bump photons

Such magnetic fields are likely to occur near the central accretion disk and, perhaps, throughout the emission line region $[7,8]$. The "clouds" in this case are likely to have a non spherical shape, perhaps elongated filaments.

One can consider also a range of properties in any given location, in a time dependent situation, where condensations or filaments are constantly produced and destroyed. Such situations are common in the interstellar medium and have also been considered in AGN. The one that attracted most attention is the locally optimally emitting cloud (LOC) model which involves an assumed range of density, column density and covering factor at any given location [18]. The various components are not in pressure equilibrium and there is little work, if any, regarding their formation or stability. However, the resulting spectral properties are quite appealing since they produce a good fit to the observed optical-UV spectrum of many AGN.

Finally we consider gas flowing away from the central disk or from other large mass reservoirs in the nucleus, in a form of a continuous wind. Such flows are characterized by continuous change of density and velocity and are very different from the above considered "clouds". The emission and absorption spectrum of such flows are very different from those produced by clouds mostly because of the different run of opacity and the structure and 
location of the various ionization fronts. The dynamics and the spectrum of AGN flows are further discussed in Subsect. 4.6.

\subsection{The Motion of Ionized Gas}

\section{The Equation of Motion}

AGN gas is exposed to a strong radiation field that can, under certain conditions, produce large scale flows. Such flows may be continuous, in which case they are classified as pure "winds", or else drive clouds or condensations in a ballistic way (the general term "wind" as used in the literature can include condensations inside a continuous flow). The general form of the equation of motion for a cloud of mass $M_{c}$ is

$$
a(r)=a_{r a d}(r)-g(r)-\frac{1}{\rho} \frac{d P}{d r}+f_{d} / M_{c},
$$

where $g(r)$ is the gravity, $a_{\text {rad }}(r)$ the acceleration due to radiation pressure and $f_{d}$ the drag force. For pure wind flows we neglect the drag force term and the internal radiation pressure. This gives

$$
v \frac{d v}{d r}=a_{r a d}(r)-g(r)-\frac{1}{\rho} \frac{d P_{g}}{d r} .
$$

An additional required equation describes the continuity condition and allows to obtain the density and velocity at every location. For the wind scenario, this is given by

$$
\dot{M}=4 \pi \rho r^{2} v C_{f}(r)=\text { const. }
$$

where $C_{f}(r)$ is the location dependent covering factor. For ballistic flows $\dot{M}$ needs to be specified more carefully, given the initial conditions, the number of moving clouds and the flow geometry.

The importance of the various terms in the equation of motion can vary a lot depending on the local conditions. Gravity dominates in almost all cases of low ionization, large column density gas. The reason is that the neutral gas at the part of the cloud away from the illuminated surface does not feel the radiation pressure force. Radiation pressure force dominates the motion of ionized, low optical depth gas near a source with a high accretion rate and large $\eta$. This is not the case for fully ionized gas where only electron scattering contributes to the pressure. Pressure gradient can be the most important factor when $a_{\text {rad }}(r) \ll g(r)$ and there is a high ionization, high temperature gas component [19].

\section{Radiation Pressure}

The radiation pressure force at a distance $r$ is obtained by considering all absorption processes and by summing over the contributions to $a_{\text {rad }}$ from all ions at that location. The contribution to $a_{\text {rad }}$ due to ion $X$ is 


$$
a_{\text {rad }}(r, X)=\frac{N_{X}}{\rho(r) c} \int_{\nu_{X}}^{\infty} \frac{L_{\nu} \chi_{\nu} e^{-\tau_{\nu}}}{4 \pi r^{2}} d \nu,
$$

where $\chi_{\nu}$ is the total absorption cross section including all bound-bound and bound-free transitions. This should be supplemented by two additional terms that count the free-free and the Compton contributions to $a_{\text {rad }}$.

The relationship between $a_{\text {rad }}$ and the level of ionization can be understood by considering the important case where the largest contribution to the radiation pressure force is due to ionization (bound-free transitions). To illustrate this we assume that the mean energy of an ionizing photon is $\overline{h \nu}$ and use the definition of $I_{X}$ from (37). For ionized gas with $N_{e} \simeq N_{H}$ this gives

$$
a_{\text {rad }}(X) \propto \frac{\overline{h \nu}}{c N_{e}} N_{X} I_{X} .
$$

In a steady state, photoionization is exactly balanced by radiative recombination, i.e. $N_{X} I_{X}=R_{X} N_{X+1} \propto N_{e}^{2}$. Thus $a_{\text {rad }}$ is proportional to $N_{X+1}$. For the specific case of hydrogen, $a_{\text {rad }} \propto N_{e}$.

It is customary to introduce the "force multiplier", $M(r)$, which is the ratio of the total radiation pressure to the Compton radiation pressure

$$
M(r)=\frac{a_{\text {rad }}(\text { total }, r)}{a_{\text {rad }}(\text { Compton }, r)},
$$

where

$$
a_{\text {rad }}(\text { Compton, } r)=\frac{N_{e}(r) \sigma_{T} L}{4 \pi r^{2} \rho(r) c} .
$$

$M(r)$ is then used to express the radiation pressure force in the equation of motion. Note that by definition $M(r) \geq 1$ and that $M(r)=1$ for fully ionized gas.

For gas which is not fully ionized, the main contributions to $M(r)$ are from bound-bound and bound-free transitions. The former dominates in those cases where the line optical depths are small. The contribution is much reduced for optically thick lines except for the illuminated surface of the cloud (or wind). The Compton and free-free terms are usually small except for the case where the gas is fully ionized. Figure 6 shows several examples of the various contributions to the force multiplier in a highly ionized AGN gas.

The gravitational and radiation pressure forces can be directly compared by writing the equation of motion in a slightly different form. This is done by noting that the mass of the central $\mathrm{BH}$ is proportional to $L_{E d d}$ and the radiation pressure force is proportional to $L$. Denoting $\Gamma=L / L_{E d d}$ and noting that $L_{E d d} \propto M_{B H}$, we obtain

$$
v \frac{d v}{d r} \simeq \frac{\sigma_{T} L}{m_{H} c 4 \pi r^{2}}[M(r)-1 / \Gamma]-\frac{1}{\rho} \frac{d P_{g}}{d r}
$$

as the equation of motion for the wind scenario. The term $M(r)-1 / \Gamma$ in this equation gives the relative importance of gravity and radiation pressure. 


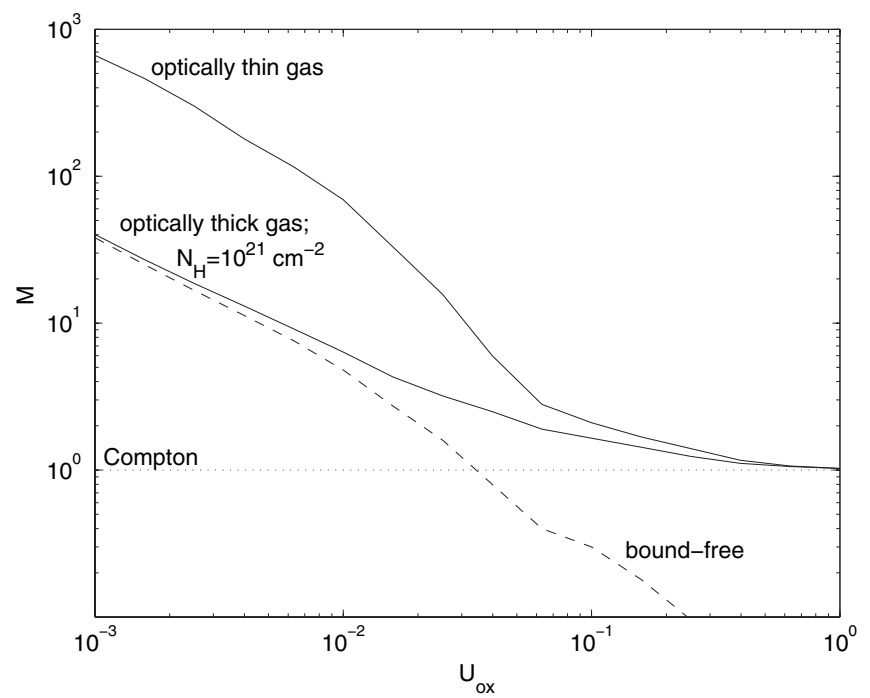

Fig. 6. The force multiplier calculated for a "standard AGN SED" and solar metallicity over a large range in ionization parameter ( $U$ (oxygen) in the case shown here). The optically thin case includes free-free, bound-free and bound-bound contribution to $M$. Bound-bound absorption dominates for all levels of ionization except for almost completely ionized gas. The large column density case is dominated by bound-free absorption since the resonance lines are very optically thick (courtesy of D. Chelouche)

Thus sources of large $\Gamma$ require smaller force multipliers to drive the wind. E.g. a source with $\Gamma=0.1$, typical of low and intermediate luminosity AGN, requires $M \geq 10$ in order to drive a wind by radiation pressure force. Such large values of $M(r)$ are normally achieved only in situations where boundbound contributions to the force multiplier are important, see Fig. 6. (Note that the constant multiplying $M(r)-1 / \Gamma$ in (59) is defined to within a factor of $\sim 1.2$ since the force multiplier and the Eddington ratio differ by the value of $\mu$, the mean molecular weight per electron. This is the reason for the use of $\simeq$ in (59)).

There are several important scenarios of radiation pressure driven flows that produce a similar asymptotic solution of the form

$$
v(r)=v_{\infty}\left[1-r_{0} / r\right]^{1 / 2},
$$

where $r_{0}$ is the launching distance of the flow and $v_{\infty}$ is the asymptotic flow velocity. For cases of constant $[M(r)-1 / \Gamma]$ we get

$$
v_{\infty} \simeq v_{c}[M-1 / \Gamma]^{1 / 2},
$$

where $v_{c}$ is proportional to $\left[L / r_{0}\right]^{1 / 2}$ and is roughly the escape velocity at the launching point of the flow. The above results are geometry dependent 
and the velocities under questions can be very different in, e.g., spherical and disk geometries. However, the launching point is a very important parameter in determining the largest obtained velocities.

The equation of motion has been solved, in various works, in an attempt to explain the velocities inferred from observations of UV and X-ray absorption lines. The first seem to indicate very high velocity flows $\left(v_{\infty}=\right.$ few $\times 10^{4}$ $\mathrm{km} \mathrm{s}^{-1}$ ) while the latter are characterized by $v_{\infty}=f e w \times 10^{2} \mathrm{~km} \mathrm{~s}^{-1}$. This can be translated to conditions near the base of the flow. For example, the maximum velocity of AGN flows that are driven by bound-free dominated radiation pressure force is of the same order as the escape velocity at the base of the flow. In such radiation pressure dominated AGN flows, the highest velocity gas must originate in the vicinity of the central accretion disk. The slower X-ray outflows can originate much further out, perhaps outside the BLR. As explained, bound-bound radiation dominated flows can reach higher velocities $\left(v_{c} \propto \sqrt{M(r)}\right)$.

\section{Main Components of AGN}

The AGN phenomenon, while defined as being in the nuclear region, can cover a large range of radii and hence a large range of physical conditions. The physical properties of any gas component are determined by its location (which determine the gravity and the ionizing flux), density (that gives the ionization parameter) and column density (specifies the optical depth and hence the effective radiation pressure force and the self-gravity). Other important factors are the local pressure (confinement), composition (opacity and emissivity) and interaction with the other components (e.g. direct or indirect line of sight to the center). The following is an attempt to specify some of those properties and investigate the observational consequences.

\subsection{The Broad Line Region}

Consider large column density $\left(\sim 10^{23} \mathrm{~cm}^{-2}\right)$ high density $\left(\sim 10^{10} \mathrm{~cm}^{-3}\right)$ gas clouds situated at a location where $L / 4 \pi r^{2} \simeq 10^{9} \mathrm{erg} \mathrm{s}^{-1} \mathrm{~cm}^{-2}$ (i.e. about 1 pc for a very luminous AGN). Consider also that the clouds can survive over many dynamical times $\left(t_{d y n} \sim 300\right.$ years in that location) either because they are confined or because they are the extensions of large self-gravitating bodies, like bloated stars. We also assume a global $(4 \pi)$ covering factor of order 0.1 .

One immediate consequence of these assumptions is that the clouds are bound, because gravity completely dominates over the radiation pressure force which is inefficient in such large column density material. The clouds will have typical velocities of order $3000 \mathrm{~km} \mathrm{~s}^{-1}$ which will be reflected in the observed widths of the emitted emission lines. 
The physical conditions in the region under discussion are such that $U$ (hydrogen $) \sim 10^{-2}$. This means that only the illuminated surface of the cloud is highly ionized. The most abundant ions in this part are He III, O IVVI, C III-IV, etc. The strongest predicted emission lines are, therefore, Ly $\alpha$, C IV $\lambda 1549$ and O vi $\lambda 1035$. The density is high but not too high to suppress all semi-forbidden lines. Strong predicted lines of this type are C III] $\lambda 1909$ and $\mathrm{O}$ III] $\lambda 1663$. As explained, much of these large column density clouds must be partly neutral since only X-ray photons can penetrate beyond an hydrogen column of $\sim 10^{22} \mathrm{~cm}^{-2}$. These parts will produce strong lines of $\mathrm{H} \mathrm{I}, \mathrm{Mg}$ II and Fe II. The observed EWs of the strongest lines depend on the emissivity and covering factor. For the specified condition they are of order 10-100 $\AA$. The observed absorption lines are extremely weak because of the small covering factor. A region with those observed properties and spectrum would definitely justify the name "broad line region" (BLR).

Can such clouds be confined? Magnetic confinement is a likely possibility since the required magnetic field is small, about $1 \mathrm{G}$. Confinement by HIM is more problematic for various reasons. The required HIM density can be obtained by the known density and temperature of the BLR gas. This gives $N_{H I M} \sim 10^{7} \mathrm{~cm}^{-3}$ where we assumed a typical Compton temperature of $10^{7} \mathrm{~K}$. Combined with the dimension of the BLR, one can obtain the total Compton optical depth that exceeds unity for the more luminous AGN. The observational consequences are clear: smearing of the central source variations and broad Compton scattering wings for all broad emission lines. This is generally not observed in AGN. There are, however, other processes that can raise the HIM temperature beyond the Compton temperature. Such relativistic HIM may still provide the required confining pressure.

Another interesting issue is the number of BLR clouds. Given the above typical velocity, and the fact that individual confined clouds are likely to emit with typical line width of $10 \mathrm{~km} \mathrm{~s}^{-1}$, we can determine the number of cloud required to produce the symmetrical smooth profiles observed in many cases. This number is huge, of order $10^{6-8}$, which raises serious question about the formation and destruction of such clouds. Alternatively, internal turbulent motion, that exceeds the sound speed by about an order of magnitude, could alleviate some of those difficulties. This needs to be justified observationally as well as theoretically but is still an open possibility.

\subsection{The Narrow Line Region}

Next we consider smaller column density $\left(\sim 10^{20-21} \mathrm{~cm}^{-2}\right)$ low density $\left(\sim 10^{4} \mathrm{~cm}^{-3}\right)$ clouds situated at a location where $L / 4 \pi r^{2} \simeq 10^{2} \mathrm{erg} \mathrm{s}^{-1} \mathrm{~cm}^{-2}$ (about $3 \mathrm{kpc}$ for a very luminous AGN). We also assume a smaller covering fraction, of order 0.01-0.1. The ionization parameter for this gas is similar to the one obtained for the BLR and the typical velocity, assuming a bound system, $500 \mathrm{~km} \mathrm{~s}^{-1}$. 
The physical conditions in this larger region are considerably different from those in the BLR, despite the very similar level of ionization. First, the column density in some of the clouds is small enough to be optically thin in the hydrogen Lyman continuum. Under such circumstances radiation pressure force may be important and the line profiles may include a nongravitational components. Optically thin gas is, on the average, more ionized than optically thick gas of the same ionization parameter. This will show in the spectrum of the gas. Confinement may not be an important issue since the life time of such large clouds is rather long. The EW of the emission lines is considerably smaller than that of the broad lines because of the smaller covering factor and the smaller Lyman continuum opacity.

The observed spectrum of this component includes intense forbidden lines, because of the low densities. This shifts the line cooling balance in such a way that the semi-forbidden and permitted lines are relatively weaker. Another group of lines that are predicted to be intense in the innermost part of this region are coronal lines, produced by fine-structure transitions and observed mostly in the infrared. The region producing such a spectrum qualifies for the name "narrow line region" (NLR).

\section{Dust and Reddening}

Dust is likely to be present in AGN emission line regions. Such dust will scatter and absorb some of the ionizing and non-ionizing radiation, and will affect the observed spectrum in various ways. There is clear evidence for dust in the NLR but no indication for dust in the BLR. The possible reasons for this, as well as for the differences in ionization and other physical conditions, are explained below.

If AGN dust is of similar composition to the dust observed in the interstellar medium of our galaxy, we can calculate a dust "sublimation radius", i.e. the minimum radius where dust of a certain composition can survive the central radiation field without evaporating. For graphite particles, this radius is given roughly by

$$
R_{s u b} \simeq 0.2 L_{46}^{1 / 2} \mathrm{pc},
$$

where $L_{46}=L / 10^{46} \mathrm{erg} \mathrm{s}^{-1}$. Reverberation mapping of the BLR shows that the mean $R_{\mathrm{BLR}}$ as determined by the emissivity of the $\mathrm{H} \beta$ line is

$$
R_{B L R} \simeq 0.4 L_{46}^{0.6 \pm 0.1} .
$$

Evidently, $R_{s u b} \simeq R_{B L R}$ which suggests that there is a natural boundary to a dust-free BLR. Since dust is such a common component in all known HII regions, it is possible that almost the entire volume of the nucleus, from just outside the BLR to the outer edge of the NLR, is filled with dust.

The presence of dust in the NLR can substantially change the level of ionization. Such dust competes, effectively, with the ionization of the gas 


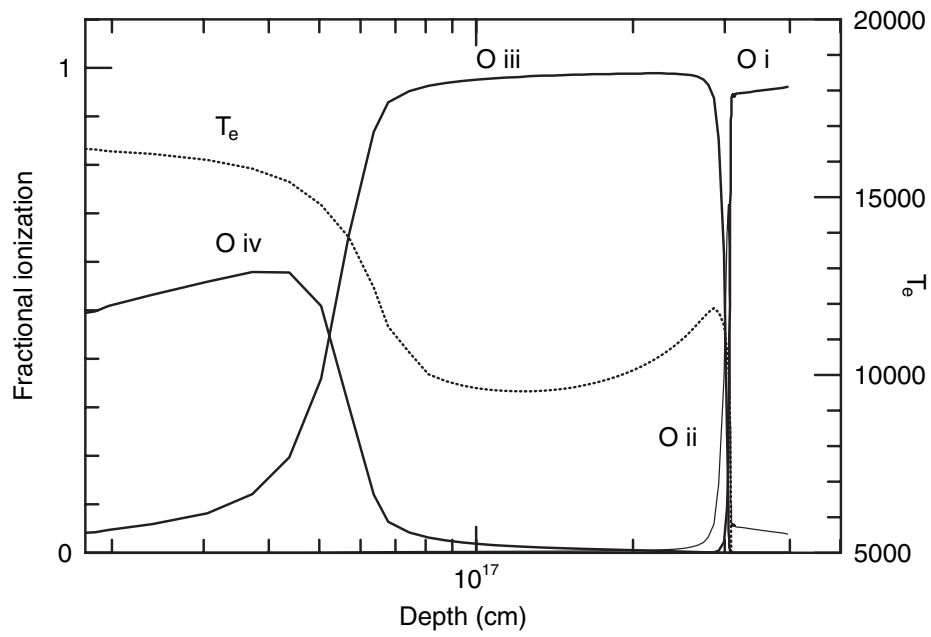

Fig. 7. Calculations by the author showing the ionization and temperature structure of a constant density dust-free gas cloud exposed to a typical AGN ionizing continuum. In this case $N_{H}=10^{4} \mathrm{~cm}^{-3}$ and $U$ (hydrogen $)=0.03$

because of its large absorption cross sections at all energies between about $0.02 \mu \mathrm{m}$ and $10 \mu \mathrm{m}$. The fraction of Lyman continuum photons absorbed by the dust, relative to those absorbed by the gas, is proportional to $U$ (hydrogen) since

$$
\left.\frac{N_{d u s t}}{N_{H^{0}}} \propto \frac{N_{\text {gas }}}{N_{H^{0}}} \propto \frac{N_{H^{+}}+N_{H^{0}}}{N_{H^{0}}} \propto U \text { (hydrogen }\right) .
$$

Therefore, dust is more efficient in attenuating the ionizing radiation in highly ionized gas, where it absorbs a larger fraction of the photons capable of ionizing hydrogen. The different ionization structures of dusty and dust-free environments are illustrated in Figs. 7 and 8.

Because of its large cross section, dust is also subjected to large radiation pressure force which is then delivered to the gas through the efficient charge coupling of the two components. In fact, the radiation pressure acceleration due to dust can be orders of magnitude larger than the acceleration given to the gas. This raises the interesting possibility that the internal pressure structure of the cloud is determined by the external radiation field through pressure balance [20]. Since outside such dusty clouds $a_{\text {rad }}(r) \propto L / r^{2}$ and inside the cloud $P_{g} \propto N_{H}$, we find that for all such clouds $L / r^{2} \propto N_{H}$. The conclusion is that for dusty ionized gas

$$
U \propto L / N_{H} r^{2}=\text { const. }
$$

This fixes both the absolute value of the ionization parameter and the fact that it is location independent. Recent dusty NLR models based on this idea are quite successful in reproducing the observed NLR spectrum while providing the physical justification for the specific observed value of $U$ (hydrogen). 


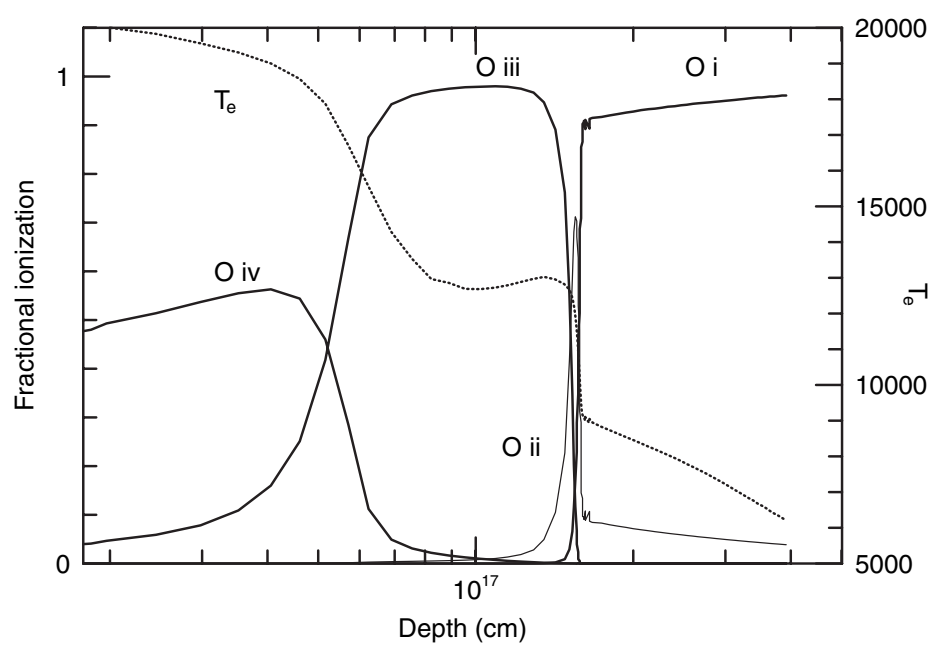

Fig. 8. Similar to Fig. 7 except that galactic type dust with the galactic dust-togas ratio is assumed to be present. Note the large reduction in the dimension of the ionized region and the increase in electron temperature mostly because of the depletion of carbon and oxygen that provide the strongest cooling lines

The NLR is, perhaps, the best observed region in active galaxies. We can therefore infer the physical conditions with great accuracy using standard line ratio techniques. We can also deduce the reddening corrections by comparing reddening sensitive line pairs. This may be somewhat tricky regarding internal dust (dust which is mixed in with the ionized gas) because of the source geometry and the distribution of the dust opacity relative to the line formation region.

There are notable exceptions to the dust-regulated constant $U$ model. Some mid-IR emission lines (e.g. [Nevi] $7.66 \mu \mathrm{m}$ ) as well as optical lines due to Fex, Fe XI and Fe XIV, clearly originate in clouds of very high level of ionization that are situated in the general NLR. These coronal lines are probably ionized by the same central continuum, but the ionization parameter is clearly much higher. It is possible that the gas producing those lines is density bounded (i.e. of low Lyman continuum optical depth) or is dust-free. In several well studied cases, there are clear signs that this part of the NLR is closer to the central source and is characterized by higher than average density and velocity. Thus, in at least several sources, the NLR is stratified with both density and ionization decreasing outward.

\subsection{The Highly Ionized Gas}

Next consider the region between the NLR and the BLR, 0.1-10 pc from the center. Assume intermediate to large $\left(10^{21-23} \mathrm{~cm}^{-2}\right)$ column density gas and a density such that the ionization parameter is 10-100 times larger than in 
the BLR. For this gas $U$ (oxygen) $\sim 0.02$ thus we expect strong absorption and emission features in the X-ray part of the spectrum. Similar temperatures and levels of ionization can be found in gas with a much lower density which is distributed inside the NLR. A proper name for such a component is the highly ionized gas (HIG). In the X-ray literature it is often called "warm absorber" [14]. For scaling purposes we note that the density of a typical HIG cloud is

$$
N_{H I G} \simeq 2 \times 10^{4} U(\text { oxygen })^{-1} L_{44}(\text { oxygen }) R_{\mathrm{pc}}^{-2} \mathrm{~cm}^{-3},
$$

and its mass is

$$
M_{\mathrm{HIG}} \simeq 10^{3} N_{22} C_{f} R_{\mathrm{pc}}^{2} M_{\odot},
$$

where $\mathrm{N}_{22}$ is the hydrogen column density (assuming solar composition) in units of $10^{22} \mathrm{~cm}^{-2}$ and $L_{44}$ (oxygen) the $0.54-10 \mathrm{X}$-ray luminosity in units of $10^{44} \mathrm{erg} \mathrm{s}^{-1}$ (for low luminosity AGN, like NGC 5548 and NGC 3783, $L_{44}($ oxygen $) \simeq 0.05$ and for high luminosity quasars $L_{44}($ oxygen $\left.) \simeq 1\right)$.

Modern X-ray observations of type-I AGN show the clear signature of such gas [21]. The strongest spectral features are numerous absorption lines of the most abundant elements, strong bound-free absorption edges due mostly to $\mathrm{O}$ VII and O VIII, and several emission lines. Perhaps the best spectrum of this type (correct to 2004) is a $900 \mathrm{ks}$ spectrum of NGC 3783 obtained by Chandra HETG. This spectrum was observed in two states of the source, high and low, that differs by about a factor of 1.5 in flux of the hard X-ray continuum. Part of the low-state spectrum is shown in Fig. 9.

The X-ray spectrum of type-II AGN is completely different, because of the large obscuration in this type of sources. It shows prominent X-ray emission lines that indicate similar properties to the gas producing the strong absorption in type-I AGN. In some of the sources the X-ray emitter is resolved and its dimension can be directly measured. This indicates X-ray emission region of several hundred pc in diameter. It is not yet clear whether the type-I absorbers are of similar dimensions. In fact, some well studied cases indicate much smaller absorption regions.

\section{HIG Outflows}

The HIG gas is transparent to the UV and soft X-ray radiation and is thus subjected to a strong radiation pressure force, compared with the opaque BLR gas. This can result in mass outflow from the nucleus. Such outflows have been observed in several well studied cases and it is interesting to consider their effects on the general AGN environment.

The mass outflow rate of the HIG can be inferred from its observed velocity and from the known (or guessed in some cases) location. It is given by

$$
\dot{M}_{H I G}=4 \pi r^{2} C_{f} \rho v_{H I G} \epsilon_{H I G}
$$




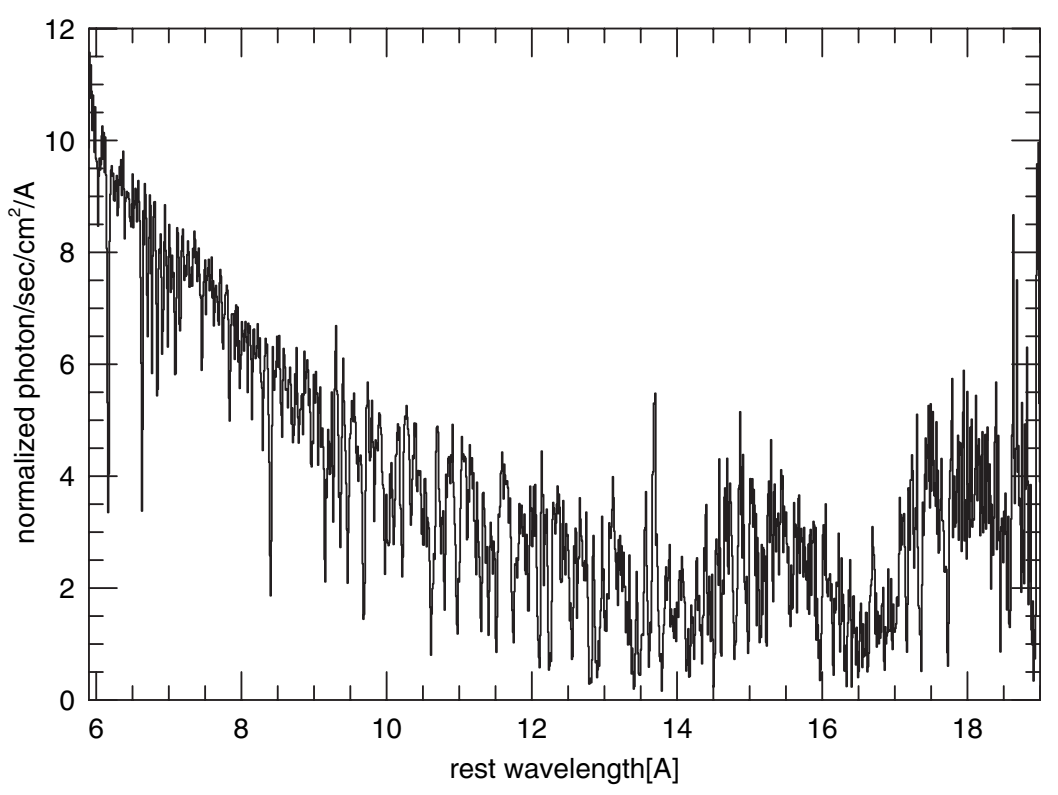

Fig. 9. Part of the low-state spectrum of NGC 3783 observed by Chandra in 2002 [22]. Some $60 \mathrm{X}$-ray lines were identified in this part of the spectrum

where $C_{f}$ is the covering fraction and $\epsilon_{H I G}$ the filling factor of the HIG. The associated dynamical time is $r_{H I G} / v_{H I G}$ which is of order a thousand years for HIG at several pc from the center and outflow velocity of several hundred $\mathrm{km} \mathrm{s}^{-1}$. Such outflows can interact with the NLR gas and disturb or modify the gas distribution at that location. It can also reach the ISM and the galactic halo on time scales of several million years with important consequences to the overall cooling and energy output to the inter-galactic medium.

\section{Associated UV Absorbers}

Are X-ray absorption lines the only signature of HIG at the above mentioned location? The answer to this question depends on the level of ionization and the column density of the gas [14]. Large columns and low ionization parameters can result in HIG whose less ionized species may well be Civ, $\mathrm{Nv}, \mathrm{O} v$ and O vi. Relatively small column density of such ions along the line of sight can produce strong UV absorption lines with typical widths similar to the widths of the X-ray absorption lines. Interestingly, some of the ions, e.g. O VI, show both UV and X-ray lines which can be compared to test the idea that the UV and X-ray absorption lines originate in the same moving gas component. Figure 11 shows a typical example of UV absorbers in an AGN whose X-ray spectrum shows the clear absorption signature of HIG. 


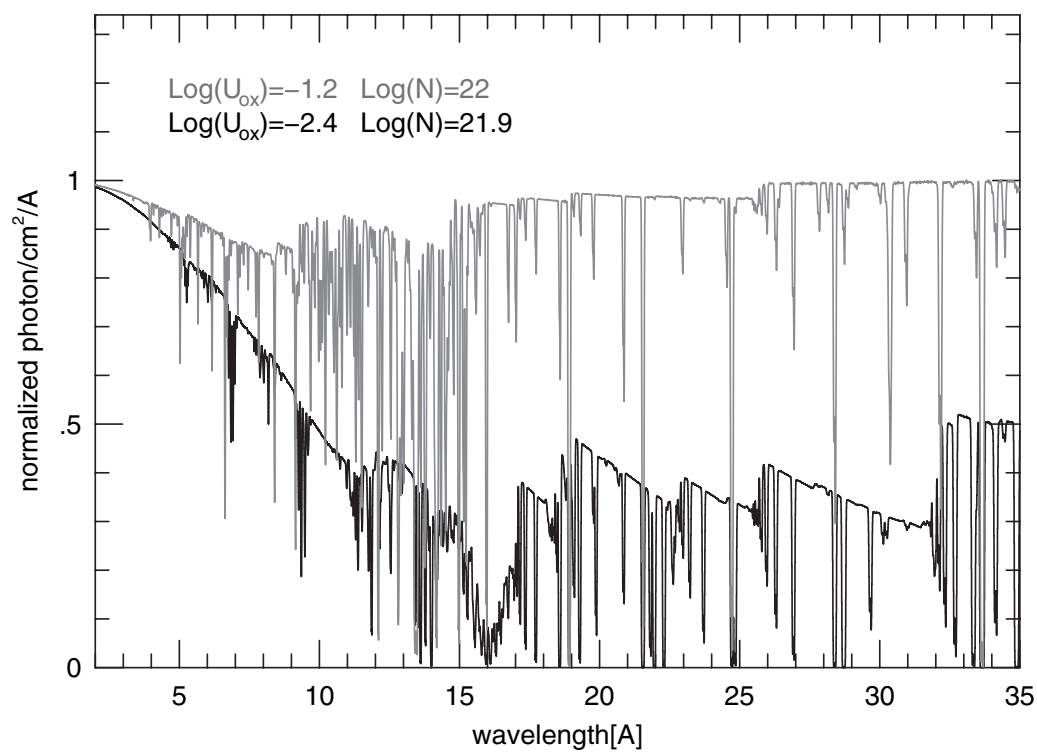

Fig. 10. Two of the three components required to model the low-state spectrum of NGC 3783 shown in Fig. 9 [23]

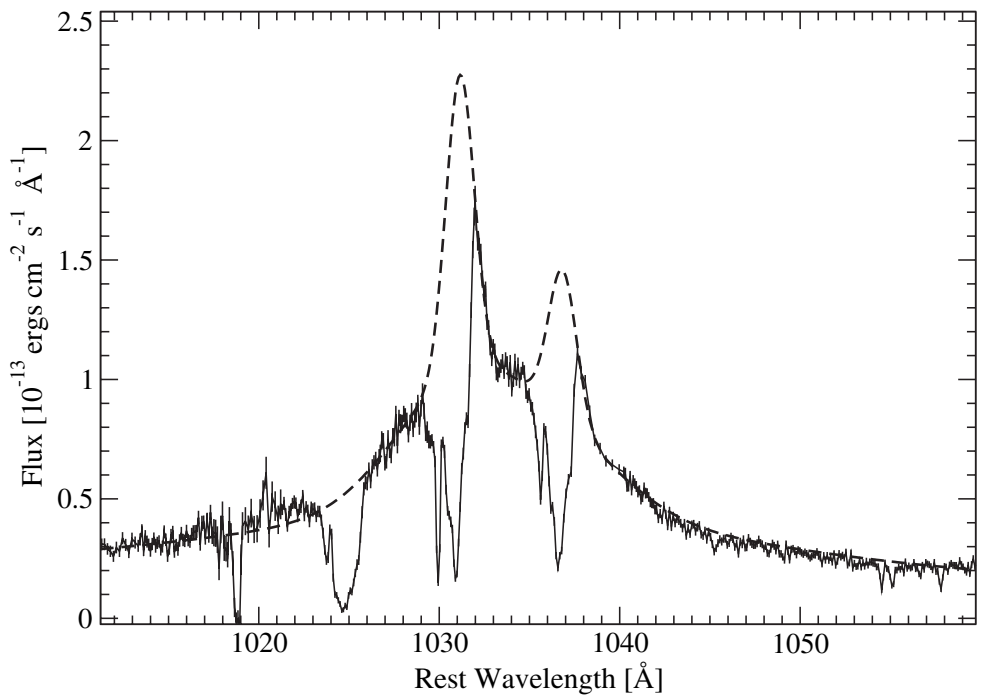

Fig. 11. The FUSE spectrum of the quasar MR 2251-178 showing narrow OvI absorption lines superimposed on emission lines of the same ion [24]. The derived outflow velocities are in the range $300-600 \mathrm{~km} \mathrm{~s}^{-1}$. The source also shows timedependent X-ray absorption lines 


\subsection{The Torus}

We next consider a region of size 1-100 pc located around the central $\mathrm{BH}$ with density of $10^{4}-10^{6} \mathrm{~cm}^{-3}$ and extremely large column density, $10^{25}$ $\mathrm{cm}^{-2}$ and even larger. We assume a flat geometry i.e. a torus or a collection of large column density clouds moving in a plane.

The above structure covers such a large range in radii that we expect a large range in physical conditions. Far from the center, at 10 or even 100 pc, the gas is very optically thick with very low temperature. Only the hard Xray radiation can penetrate that far and even those photons are limited to a few Compton depths. The conditions must be similar to those in molecular gas in the galaxy. Such regions are likely to contain large amount of dust. Their spectral signature are infrared emission and absorption with strong dust features.

The inner part of such a structure is exposed to the central radiation field. The temperature and ionization of this part depend on the ionization parameter. Using the previously defined $L_{44}$ (oxygen) and assuming a typical $\mathrm{X}$-ray SED we estimate that

$$
U(\text { oxygen }) \simeq 0.2 L_{44}(\text { oxygen }) r_{p c}^{-2} N_{5}^{-1},
$$

where $N_{5}=N_{H} / 10^{5} \mathrm{~cm}^{-3}$ and $r_{p c}$ the distance to the inner wall in pc. Assume for example $L_{44}$ (oxygen) $=0.1$ (intermediate luminosity AGN), $r_{p c}=1$ and $N_{5}=0.1$. This gives $U$ (oxygen) $=0.2$. This would produce strong high ionization X-ray lines from the torus cavity. In particular, low, intermediate and high ionization iron $K_{\alpha}$ lines are expected to show as a clear signature of such a structure. Because of the huge column density, such a wall is a very efficient "X-ray reflector" which will scatter and reflect the incident X-ray continuum radiation. Finally, we note that the inner parts of such a torus are a likely gas reservoir for both X-ray driven winds and accretion onto the central BH.

\subsection{Stars and Starburst Regions}

We next consider stars and starburst regions at various locations.

Stars in the (hypothetical) nuclear cluster can absorb and reprocess the incident AGN continuum. Regarding main sequence stars, those are not likely to show any spectral signature because of the high density atmospheres and the small cross section (i.e. small covering factor). However, some stars may develop extended winds, or envelops, as a result of the interaction of their atmosphere with the hard, external radiation field. This will lower the density and increase the cross section for absorbing the ionizing radiation. Such extended envelope stars have been named "bloated stars". It has been speculated that star-star collisions may also produce bloated stars.

Bloated stars extended envelopes are similar, in many ways, to the low density gas discussed so far. Close to the center they will show typical BLR 
spectrum and further away a typical NLR spectrum. Moreover, such winds are likely to have a large density gradient that, far enough from the stars, could be observed as typical HIG. Thus the presence of giant envelope stars can bridge the gap between several observed components of AGN with different densities [25]. This can also provide another reservoir, or source, for the emission line gas.

Starburst regions are normally considered as a different component of AGN. The typical dimension of such regions is several $\mathrm{kpc}$ and the typical density $10^{0}-10^{3} \mathrm{~cm}^{-3}$. Such regions contain their own internal source of mechanical energy: supernovae explosions and fast stellar winds. The typical X-ray signature of starburst regions is that of a multi-temperature hot plasma, i.e. strong collisionally excited emission lines. An example is given in Fig. 12 which shows the X-ray spectrum of the typical starburst galaxy M82.
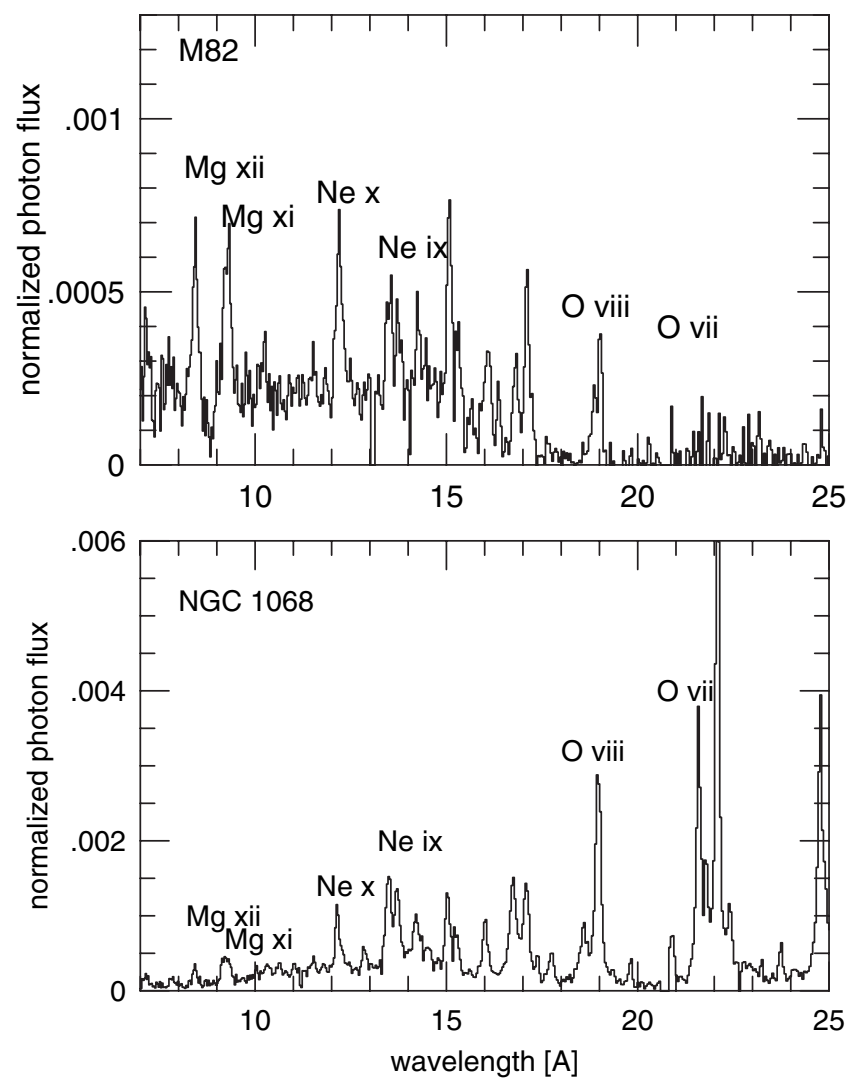

Fig. 12. A comparison of X-ray photoionized gas (NGC 1068, a type-II AGN with an extended X-ray nebulosity) and X-ray collisional gas (M82, a "classical" starburst galaxy). Both spectra were obtained by the RGS on board XMM-Newton and were retrieved from the XMM archive 
For comparison we also show the X-ray spectrum of the "classical" type-II AGN, NGC 1068, obtained with the same instrument. The two spectra are, indeed, very different and the relative emission line ratios clearly show the different excitation mechanisms.

Close enough to the central source, the starburst excited gas is also exposed to the strong, hard radiation field. Depending on the density and flux, the resulting ionization parameter can be high enough to cause significant additional ionization thus changing the ionization balance and the emergent spectrum. We expect such starburst regions to show a mixed-type spectrum which can differ a lot from the spectrum of starburst regions that are not exposed to a central radiation source.

\subsection{The Central Accretion Disk}

Finally, consider the central accretion disk itself. This is an object of high density, large column density gas at 10-100 $r_{g}$ which can be exposed to part of the centrally produced continuum emission. This can result in emission and/or absorption lines, depending on the geometry.

The term "central radiation field" should be defined more carefully since, so far, it has been used to describe the radiation emitted by material in the disk and in its vicinity. The disk itself, i.e. the part emitting the opticalUV radiation, is exposed to only some of the emitted SED, e.g. the X-ray radiation. The exact geometry is, therefore, of paramount importance. Plausible models considered in the literature include the possibility that the disk is illuminated by a central point source, by a double X-ray source (i.e. two point sources above and below the plane of the disk) and by the X-rays produced and scattered by an extended corona [26]. The unknown geometry, plus the fact that the density of the disk depends on the uncertain value of the parameter $\alpha$, leave a large range of acceptable ionization parameters.

The results of such a scenario are easy to predict. For a low ionization parameter, the main spectral signatures are fluorescence lines of relatively neutral species and a strong reflected X-ray continuum. The strongest lines are due to FeI-XVII, at around $6.4 \mathrm{keV}$, because of the combination of high abundance and large fluorescence yield for such ions. For higher ionization parameter, the "skin" of the disk is ionized and is a source of high excitation high ionization X-ray lines. The strongest predicted lines are the leading resonance transitions in the $\mathrm{H}$-like and He-like sequences of carbon, nitrogen, oxygen, neon silicon and iron. Such disk lines are predicted to be broadened by the strong gravitational field, and by the large rotational velocity of the gas, if produced close enough to the central $\mathrm{BH}$.

To summarize all these possibilities we show in Table 2 a list of all possible locations considered here with the known or expected density and the corresponding ionization parameters. 
Table 2. AGN components

\begin{tabular}{llll}
\hline Component & Location & Density & Ionization Parameter \\
\hline Accretion disk & $10^{-3} \mathrm{pc}$ & $\sim 10^{15} \mathrm{~cm}^{-3}$ & $U$ (oxygen) $=10^{-3}-10^{-1}$ \\
BLR & $0.01-1 \mathrm{pc}$ & $\sim 10^{10} \mathrm{~cm}^{-3}$ & $U$ (hydrogen) $\sim 10^{-2}$ \\
Torus & $1-10 \mathrm{pc}$ & $10^{3-6} \mathrm{~cm}^{-3}$ & $U$ (oxygen) $\sim 10^{-2}$ \\
HIG & $\sim 1 \mathrm{pc}$ & $10^{3}-10^{5} \mathrm{~cm}^{-3}$ & $U$ (oxygen) $\sim 10^{-2}$ \\
NLR & $100-1000 \mathrm{pc}$ & $10^{3-5} \mathrm{~cm}^{-3}$ & $U$ (hydrogen) $\sim 10^{-2}$ \\
Starburst & $\sim 1000 \mathrm{pc}$ & $10^{0}-10^{3} \mathrm{~cm}^{-3}$ & $U$ (oxygen) $=10^{-2}-10^{-4}$ \\
\hline
\end{tabular}

\subsection{AGN Winds}

Can all or most of these components be combined into a simplified model where the various locations, and the different dynamics, form a single, more coherent picture? Such ideas have been proposed in recent years [27]. The general picture is that of a "wind model" that originates from the vicinity of the central BH, perhaps from the surface of the accretion disk. The components are linked to this global picture, some with more justification than others. The more important ingredients of the model are:

Disk wind: Such winds are assumed to be lifted along the magnetic field lines as shown in Fig. 3. They can also be evaporated from the disk upper surface in a vertical direction. The gas gets an additional "kick" at some height above the disk surface, where it is first exposed to part or all of the central radiation. The flow accelerates due to radiation pressure force and moves to large distances. The flow lines can change direction due to the combined effect of all forces and the global velocity field is rather different from a simple spherical outflow.

Broad and narrow absorption lines: These are most naturally explained by the wind model. They are observed to be broad or narrow depending on the location and the line-of-sight to the center. An important factor for disk winds is the amount of spectral shielding since very high velocity outflows require shielding at their base. The spectral appearance of such flows is very sensitive to the observers' line-of-sight and can produce rather different appearances at different viewing angles. Many absorption lines are optically thick yet they are not black at their center since the covering factor along some viewing angles is less than unity.

Broad emission lines: The BLR in the wind scenario is assumed to be made of high density, optically thick condensations (or regions) in the wind. This is a weak point of the model since no calculation can currently produce all the observed properties of this region. Moreover, there are some indications, based on reverberation studies, of Keplerian (random orientation) velocities in the BLR of several well studied objects. 
Narrow emission lines: Naive arguments suggest that the outflow can reach the NLR and form larger condensations. There are no calculations that support this claim.

HIG outflows: This is, perhaps, the most successful aspect of the model since highly ionized X-ray gas is easy to produce in many different situations. However, the observed velocities of such gas are an order of magnitude smaller than the escape velocity from the disk.

Mass loss rate: The required mass loss rate in most global wind models considered so far is of the same order as the mass accretion rate to the $\mathrm{BH}$.

Global AGN wind models are only just starting to emerge. It remains to be seen whether the more complete calculations can explain the observed properties of AGN, given such winds.

\section{Summary}

The basic physics of the central power house in AGN must be related to accretion disks around massive BHs. A well developed theory of such structures, in particular of optically thick geometrically thin accretion disks, helps to understand the optical-UV continuum of AGN. The observed strong X-ray source is likely to originate in a separate component, such as a disk corona. The emergent continuum SED, together with the density, column density and the location of the gas relative to the $\mathrm{BH}$, determine the physical conditions and the emission and absorption spectra of such objects. Examples that were treated here in some detail include gas in the BLR, in the NLR, the HIG, bloated stars, gas in star forming regions and the central accretion disk.

\section{Acknowledgements}

This work is supported by the Israel Science Foundation and by the Jack Adler Chair of Extragalactic astronomy.

\section{References}

1. Barkana, R., \& Loeb, A., 2001, Phys. Rep, 349, 125

2. Krolik, J.H., "Active Galactic Nuclei" (Princeton Series in Astrophysics)

3. Shakura, N., \& Sunyaev, R., 1973 (A\&A 24, 337)

4. Frank, King \& Raine "Accretion Power in Astrophysics" (Cambridge University Press)

5. Blandford, R., 1990 in "Active Galactic Nuclei" (Saas-Fee 1990)

6. Laor, A., \& Netzer, H., 1989, MNRAS, 238, 897

7. Konigl, A. \& Kartje, J., 1994, ApJ, 434, 446

8. Emmering, R.T., Blandford, R.D., \& Shlosman, I. 1992, ApJ, 385, 460 
9. Balbus, S.A., \& Hawley, J.F., 2003, LNP Vol. 614: Turbulence and Magnetic Fields in Astrophysics, 614, 329

10. Narayan, R. 2005, Astrophys.Space.Science 300, 177

11. Liu, B.F., Mineshige, S., \& Ohsuga, K., 2003, ApJ 587, 571

12. Netzer, H. 1990, in "Active Galactic Nuclei" (Saas-Fee 1990)

13. Peterson, B., "Introduction to Active Galactic Nuclei" (Cambridge University Press)

14. Crenshaw, Kraemer \& George, I., 2003, AnnRevAstAP, 41, 117

15. Ferland, G., 2003, AnnRevAstAp, 41, 517

16. Krolik, J.H., McKee, C.F., \& Tarter, C.B., 1981, ApJ, 249, 422

17. Rees, M.J., Netzer, H., \& Ferland, G.J., 1989, ApJ, 347, 640

18. Baldwin, J., Korista, K., \& Ferland, G., 1997, ApJ, 487, 555

19. Chelouche, D., \& Netzer, H., 2005, ApJ, 625, 95

20. Groves, B.A., Dopita, M.A., \& Sutherland, R.S., 2004, ApJS, 153, 9

21. Paerels, F.B.S., \& Kahn, S.M., 2003, AnnRevAstAp, 41, 291

22. Kaspi, S., et al. 2002, ApJ, 574, 643

23. Netzer, H., et al. 2003, ApJ, 599, 933

24. Kaspi, S., Netzer, H., Chelouche, D., George, I.M., Nandra, K., \& Turner, T.J. 2004, ApJ, 611, 68

25. Alexander, T., \& Netzer, H., 1997, MNRAS, 284, 967

26. Fabian, A.C., 2005, Astrpy. SS, 300, 97

27. Elvis, M., 2000, ApJ, 545, 63 